Fl. Medit. 26: 31-61

doi: 10.7320 /FlMedit26.031

Version of Record published online on 08 September 2016

\title{
Evagelos Baliousis
}

\section{Flora and vegetation of Mt Aphrodisio (Peloponnisos, Greece)}

\begin{abstract}
Baliousis, E.: Flora and vegetation of Mt Aphrodisio (Peloponnisos, Greece). - Fl. Medit. 26: 31-61. 2016. - ISSN: 1120-4052 printed, 2240-4538 online.

This paper presents 650 specific and infraspecific taxa of the vascular flora of Mt Aphrodisio, NW Peloponnisos. All the records are new as the mountain was until now floristically unexplored. For each taxon, local distribution and habitat types are presented. Crypsis alopecuroides is new record for Peloponnisos. Its flora comprises also 29 Greek and 22 Balkan endemics. Some of the records concern rare taxa in Greece, in Peloponnisos or regional endemics, which are, therefore, chorologically significant, such as Arenaria guicciardii, Alkanna methanaea, Erysimum pectinatum, Anthemis brachmannii, Silene gigantea subsp. hellenica, Delphinium hellenicum, Galium capitatum, Verbascum daenzeri, Trifolium tenuifolium, Glinus lotoides, Helleborus odorus subsp. cyclophyllus, Galanthus reginae-olgae subsp. vernalis, Bromus parvispiculatus, Crypsis schoenoides, Gaudiniopsis macra. The main vegetation types are also described.
\end{abstract}

Key words: biodiversity, phytogeography, vegetation, Peloponnisos, Mediterranean.

\section{Introduction}

Mt Aphrodisio is located at the northwestern part of Peloponnisos and belongs to the homonymous unit according to the phytogeographical division in "Flora Hellenica" (Strid \& Tan 1997). More specifically it constitutes the border between the prefectures of Arkadia and Achaia in contact with the prefecture of Ilia. Its name is derived by the sanctuary of goddess Aphrodite situated on a saddle-shaped pass between the main peeks of the mountain at $1150 \mathrm{~m}$.

The main peaks of Mt Aphrodisio (Fig. 1), Mavri Vrisi (1445 m) and Neraidorrachi $(1369 \mathrm{~m})$ are located in its main axis which has a NW-SE orientation. Northern and western boundaries of the investigated area are clearly demarcated by the rivers Seiraios and Erimanthos respectively. Southern foothills of the mountain are crossed by the river Ladonas from the homonymous artificial lake to the hydroelectric power plant near the village Kato Spatharis. Eastward the slopes of the mountain descend to small plains around the village Dafni. 
Geologically, the investigated area belongs to the geotectonic unit of Olonos-Pindos. The main substrates are limestones followed by radiolarites and flysch. Longitudinal zones of these types of rocks mainly with SW-NE orientation continuously succeed each other in the whole area. Talus cones are restricted to small areas in the slopes which descend to rivers Ladonas, Erimanthos and Seiraios. Alluvial deposits occupy a narrow strip of land formed by Seiraios river in the north. Conglomerates cover a relatively extensive zone south of the village Voutsis (IGME 1978). In general the geological landscape is quite fragmented and this is sometimes reflected in the distribution pattern of the various types of

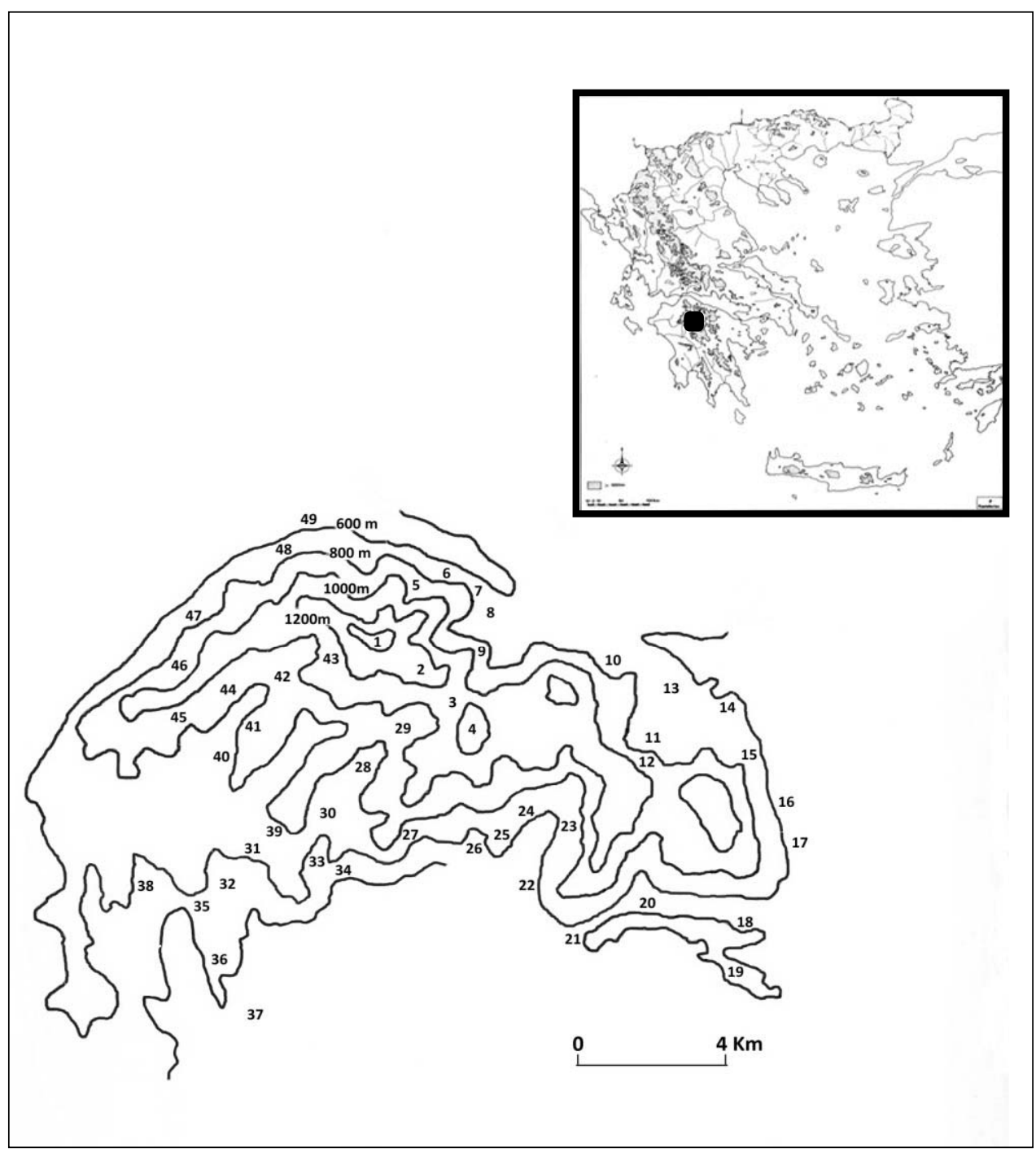

Fig. 1. Geographical position of Mt Aphrodisio in Greece and a map of the investigated area. 
vegetation. The maximum boundaries of the investigated area are defined by the coordinates $37^{\circ} 44^{\prime} 48^{\prime \prime}$ to $37^{\circ} 51^{\prime} 54^{\prime \prime} \mathrm{N}$ and $21^{\circ} 47^{\prime} 40^{\prime \prime}$ to $22^{\circ} 03^{\prime} 25^{\prime \prime} \mathrm{E}$.

The nearest meteorological station which provides full climatic data is that of Kalavrita situated at $731 \mathrm{~m}$. According to climatic diagram by Emberger $(1955,1959)$ and Sauvage (1963), the bioclimate of the area is humid with cool winter. The dry period, according to the ombrothermic diagram by Bagnouls \& Gaussen (1957) lasts approximately four months. The mean annual height of precipitation in the meteorological stations of Tripotama $(550 \mathrm{~m})$ and Dafni $(580 \mathrm{~m})$ situated at the northern parts of the investigated area reaches approximately $1000 \mathrm{~mm}$. Regional and local differences exist depending on altitude and topography.

Human intervention on the ecosystems of the mountain is on decline nowadays. A few decades ago the area was relatively densely populated with numerous villages dispersed in its lower altitudinal zone. The main occupation of the inhabitants was raising of sheep and goats in combination with the cultivation of the low productivity stony terrain. Arkadia was famous since antiquity for its pastures where the god Panas acted protecting the breeders. Livestock breeding still remains the base of the local economy but its size has dramatically reduced since it is practiced by a few elderly people. The majority of the villages look nowadays abandoned or inhabited by few people. Most of the productive age people abandon the area seeking professional occupation in the biggest cities of Peloponissos and in Athens. Thus the area is nowadays sparsely populated and many villages are lacking of young generation. There is also no prospect of touristic development as in other areas of Arkadia. As this situation seems irreversible the future of the local ecosystems is expected to be prosperous. Natural vegetation dominated by Quercus coccifera has already taken over abandoned anthropogenic habitats such as stony fields.

Aphrodisio is one of the few floristically unexplored mountains of Greece. Bibliographical data concerning previous reports are lacking and that stimulated my interest to conduct this study. The aim of this study is also to reveal the vascular plant diversity of medium sized mountains of Greece which are less investigated as compared to higher massifs.

\section{Material and methods}

The study is based on collections and field observations made from 2005 to 2013. Collections were conducted in various localities and habitats of the mountain in all the seasons of the year in order to obtain a precise idea of the character of its flora. All specimens, about 1500, are temporarily kept in my personal herbarium. Species identification and/or nomenclature were based mostly on Davis (1965-1985), Tutin \& al. (1968, 1972, 1976, 1980, 1993), Greuter \& al. (1984, 1986, 1989), Strid \& Tan (1997, 2002), Greuter \& RaabStraube (2008) and Dimopoulos \& al. (2013). Families, genera, species and subspecies are listed within the major taxonomic groups in alphabetical order. In the following catalogue only spontaneous and subspontaneous taxa are recorded. Transliteration of localities is in accordance with “Flora Hellenica” (Strid \& Tan 1997, 2002). 


\section{Localities (Fig. 1)}

1. Summit Mavri Vrisi, $1445 \mathrm{~m}, 21.6 .2005$.

2. Between summit Mavri Vrisi and the sanctuary of Aphrodite, 1150-1300 m, 14.5.2015, 5.6.2005, 28.3.2006, 25.6.2006.

3. Sanctuary of Aphrodite, $1150 \mathrm{~m}, 14.5 .2015$, 5.6.2005, 28.3.2006, 25.6.2006, 25.5.2013.

4. Summit Neraidorrachi, $1150-1369 \mathrm{~m}, 25.5 .2013$.

5. c. $0.5 \mathrm{~km} \mathrm{SE}$ of Dechouni village, $850 \mathrm{~m}, 24.5 .2013$.

6. Between Dechouni and Dechouneika villages, $700 \mathrm{~m}, 24.5 .2013$.

7. Dechouneika village, $620 \mathrm{~m}, 24.5 .2013$.

8. Crossroad to Kondovazena and Dechouni villages, $620 \mathrm{~m}, 24.5 .2013$.

9. Between Dechouneika village and the sanctuary of Aphrodite, 700-900 m, 26.5.2013.

10. c. $0.5 \mathrm{~km} \mathrm{~N}$ of Vesini village, $700 \mathrm{~m}, 26.5 .2013$.

11. Nasia village, $700 \mathrm{~m}, 26.5 .2013$.

12. c. $1 \mathrm{~km} \mathrm{~S}$ of Nasia village, $700-800 \mathrm{~m}, 26.5 .2013$.

13. Between Nasia and the crossroad to Dafni, 550-700 m, 26.5.2013.

14. Between Dafni and the crossroad to Nasia, 550-600 m, 25.5.2013, 26.5.2013.

15. Dafni village, $600 \mathrm{~m}, 24-26.5 .2013$.

16. Between Dafni and Pournaria village, 500-600 m, 25.5.2013.

17. Pournaria village, $520 \mathrm{~m}, 25.5 .2013$.

18. Mouria village, $460 \mathrm{~m}, 25.5 .2013$.

19. Eastern shores of Ladona artificial lake, $420 \mathrm{~m}, 14.10 .2005$, 18.6.2006, 24.6.2006.

20. Between Mouria village and the dam of Ladona lake, 430-460 m, 25.5.2013.

21. Dam of Ladona lake, $430 \mathrm{~m}, 25.5 .2013$.

22. Between Pera Vachlia village and the dam of Ladona lake, 430-600 m, 25.5.2013.

23. Pera Vachlia village, $600 \mathrm{~m}, 25.5 .2013$.

24. Vachlia village, $620 \mathrm{~m}, 25.5 .2013$.

25. Between Vachlia and Dimitra villages, 540-620 m, 25.5.2013.

26. Dimitra village, $540 \mathrm{~m}, 25.5 .2013$.

27. Between Kondovazena and Dimitra villages, 540-700 m, 25.5.2013.

28. Kondovazena village, $700 \mathrm{~m}, 11.10 .2005,22.2 .2006,27.3 .2006,25.6 .2006$, 25.5.2013, 26.5.2013.

29. Between Kondovazena and the sanctuary of Aphrodite, 700-1100 m, 25.6.2006, 25.5.2013.

30. Between Kondovazena and the crossroad to Peleki village, 600-700 m, 11.10.2005, 22.2.2006, 27.3.2006, 25.6.2006, 19.4.2012, 25.5.2013.

31. Crossroad to Peleki village, $600 \mathrm{~m}, 19.4 .2012$.

32. Between the crossroad to Peleki and Voutsis villages, 550-600 m, 14.5.2005, 5.6.2005, 25.6.2006, 19.4.2012.

33. Between the main road and the monastery of Klivoka, 450-630 m, 25.5.2013.

34. The monastery of Klivoka, $450 \mathrm{~m}, 22.2 .2006,25.5 .2013$.

35. Voutsis village, $550 \mathrm{~m}, 19.4 .2012$.

36. Between Voutsis village and the hydroelectric power plant near Kato Spatharis village, 210-550 m, 14.5.2005, 19.4.2012. 
37. Hydroelectric power plant near Kato Spatharis village, 210 m, 14.5.2005, 5.6.2005, 25.6.2006, 19.4.2012.

38. Monastiraki village, $600 \mathrm{~m}, 19.4 .2012$.

39. Southern slopes of Koprisies summit, 650-750 m, 19.4.2012.

40. c. $1.5 \mathrm{~km} \mathrm{~S}$ of Aposkia village, $800 \mathrm{~m}, 19.4 .2012$.

41. Aposkia village, $840 \mathrm{~m}, 19.4 .2012$.

42. c. $0.5 \mathrm{~km}$ NE of Soudeli settlement, $850 \mathrm{~m}, 21.6 .2005$.

43. Between Soudeli and the summit of Mavri Vrisi, 900-1400 m, 21.6.2005.

44. Velimachi village, $860 \mathrm{~m}, 19.4 .2012$.

45. Kardaritsi village, $900 \mathrm{~m}, 19.4 .2012$.

46. Between Kardaritsi and Paralogi villages, 800-900 m, 19.4.2012.

47. Paralogi village, $800 \mathrm{~m}, 19.4 .2012$.

48. c. $1 \mathrm{~km} \mathrm{~S}$ of Tripotama village, $600 \mathrm{~m}, 19.4 .2012,24.5 .2013$.

49. Tripotama village, $540 \mathrm{~m}, 19.4 .2012,24.5 .2013$.

\section{Habitats}

a. Quercus coccifera dominated scrub often mixed with scattered deciduous species such as Fraxinus ornus, Quercus pubescens, Crataegus monogyna, Acer monspessulanum subsp. monspessulanum, mainly limestone.

b. Open rocky places, limestone.

c. Stony meadows with scattered individuals of Quercus coccifera and Crataegus heldreichii.

d. Quercus frainetto forest, mainly radiolarites.

e. Quercus pubescens forest.

f. Quercus frainetto-Quercus pubescens mixed forest.

g. Carpinus orientalis forest.

h. Mixed deciduous forest with Quercus pubescens, Carpinus orientalis, Fraxinus ornus.

i. Limestone cliffs.

j. Macchie with Quercus coccifera, Arbutus unedo, Erica arborea, Pistacia lentiscus, Calicotome villosa.

k. Platanus orientalis forest.

1. Open grazed places.

m. Damp to wet places by springs or road margins, ditches and brooks.

n. Fallow and abandoned fields.

o. Fields (mainly olive groves and walnut orchards).

p. Forest roadsides.

q. Road cuttings.

r. Roadsides.

s. Street margins and disturbed places.

t. Stone walls and dry stone walls.

u. Muddy places by lake margins. 


\section{Plant list}

The following abbreviations are used: Bal. = E. Baliousis; obs. = field observation; phot. $=$ photograph; s.n. $=$ without number. Names of taxa not native to the investigated area are set in square brackets.

\section{PTERIDOPHYTA}

\section{ASPLENIACEAE}

Asplenium ceterach L. - 2b, Bal. 1902; 4b, Bal. 8342; 30a, Bal. s.n.

Asplenium onopteris L. - 5a, Bal. 8147; 10g, Bal. 8794.

Asplenium trichomanes L. s.l. - 2b, Bal. 1903; 6k, Bal. 8144; 5a, Bal. 8146; 10g, Bal. 8795.

\section{DENNSTAEDTIACEAE}

Pteridium aquilinum (L.) Kuhn subsp. aquilinum -42p, Bal. 2123; 2e, Bal. 2304; 48e, Bal. 8233; 36r, Bal. obs.

\section{EQUISETACEAE}

Equisetum arvense L. - 14m, Bal. 8667.

Equisetum ramosissimum Desf. - 34m, Bal. 8511.

Equisetum telmateia Ehrh. - 37k, Bal. 1781.

POLYPODIACEAE

Polypodium cambricum L. - 34i, Bal. 2232.

PTERIDACEAE

Adiantum capillus-veneris L. - 37m, Bal. s.n.

SELAGINELLACEAE

Selaginella denticulata (L.) Spring - 34a, Bal. 8522.

SPERMATOPHYTA

GYMNOSPERMAE

EPHEDRACEAE

Ephedra foeminea Forssk. - 34i, Bal. 8474.

\section{ANGIOSPERMAE \\ DICOTYLEDONES}

ACANTHACEAE

Acanthus spinosus L. - 7l, Bal. obs.; 8r, Bal. obs.; 28n, Bal. obs.; 23r, Bal. obs. 
ACERACEAE

Acer monspessulanum L. subsp. monspessulanum - 2c, Bal. 2531; 12d, Bal. 8745.

Acer sempervirens L. - 34a, Bal. 8536.

AMARANTHACEAE

[Amaranthus albus L.] - 19u, Bal. 2161.

\section{ANACARDIACEAE}

Pistacia lentiscus L. - 36j, Bal. obs.

Pistacia terebinthus L. subsp. terebinthus - 30a, Bal. 8398; 32a, Bal. obs.; 31a, Bal. obs.

\section{APIACEAE}

Anthriscus sylvestris subsp. nemorosus (M. Bieb.) Koso-Pol. -41k, Bal. 6190.

Bubon macedonicum L. - 27q, Bal. 8611.

Bupleurum glumaceum Sm. - 42p, Bal. 2099; 2c, Bal. 2544; 30n, Bal. s.n.

Chaerophyllum nodosum (L.) Crantz - 7k, Bal. 8082; 6k, Bal. 8122.

Chaerophyllum temulum L. $-7 \mathrm{~m}$, Bal. 8081.

Daucus carota subsp. maximus (Desf.) Ball - 32r, Bal. 2632.

Daucus guttatus Sm. subsp. guttatus - 32r, Bal. 2015; 7n, Bal. 8073; 33p, Bal. 8430;

30n, Bal. 8575 .

Elaeoselinum asclepium (L.) Bertol. subsp. asclepium - 9a, Bal. 8800.

Eryngium amethystinum L. - 1c, Bal. 2071; 4c, Bal. 8376.

Eryngium campestre L. - 30r, Bal. 2610; 71, Bal. obs.

Eryngium creticum Lam. - 33p, Bal. 8431.

Foeniculum vulgare Mill. - 28r, Bal. 2619; 14r, Bal. obs.

Geocaryum parnassicum (Boiss. \& Heldr.) Engstrand - 4c, Bal. 8333.

Helosciadium nodiflorum (L.) W.D.J. Koch - 7m, Bal. 8074.

Heracleum sphondylium subsp. ternatum (Velen.) Briq. - 13m, Bal. 8781.

Lagoecia cuminoides L. - 5r, Bal. 8172.

Malabaila aurea (Sm.) Boiss. - 34p, Bal. 8493.

Oenanthe pimpinelloides L. s.l. -37m, Bal. 1780.

Opopanax hispidus (Friv.) Griseb. - 34p, Bal. 8467.

Orlaya daucoides (L.) Greuter - 2c, Bal. 1981; 1c, Bal. 2052; 5a, Bal. 8178.

Orlaya grandiflora (L.) Hoffm. - 33p, Bal. 8429.

Scaligeria napiformis (Spreng.) Grande - 37r, Bal. 2023; 34p, Bal. 8524; 9a, Bal. 8801.

Scandix australis subsp. grandiflora (L.) Thell. - 2c, Bal. 1886; 5a, Bal. 8174; 4c, Bal. 8334; 8r, Bal. s.n.

Scandix pecten-veneris L. - 7n, Bal. 8093.

Smyrnium perfoliatum L. s.l. - 30n, Bal, 6155; 7n, Bal. 8075.

Tordylium apulum L. - 2c, Bal. 1887; 31r, Bal. s.n.; 7r, Bal. s.n.

Tordylium officinale L. - 7r, Bal. 8078.

Torilis africana Spreng. - 42p, Bal. 2108; 2c, Bal. 2566; 7r, Bal. 8080; 34p, Bal. 8479; 9r, Bal. 8802.

Torilis arvensis subsp. recta Jury - 37m, Bal. 2024; 28m, Bal. 2624. 
Torilis leptophylla (L.) Rchb. f. - 5r, Bal. 8173; 7n, Bal. s.n.

Torilis nodosa (L.) Gaertn. - 2c, Bal. 2008; 7n, Bal. 8079.

\section{APOCYNACEAE}

Vinca herbacea Waldst. \& Kit. - 2c, Bal. 1868; 30a, Bal. 2261; 39a, Bal. 6164.

[Vinca major L. subsp. major] - 41n, Bal. obs.; 47n, Bal. obs.

\section{ARALIACEAE}

Hedera helix L. subsp. helix - 41k, Bal. obs.

\section{ARISTOLOCHIACEAE}

Aristolochia microstoma Boiss. \& Spruner - 2c, Bal. 1866; 7n, Bal. 8110; 4c, Bal. 8366.

This is the westernmost locality of this Greek endemic.

\section{ASTERACEAE}

Achillea ligustica All. - 12p, Bal. 8760.

Achillea setacea Waldst. \& Kit. - 3c, Bal. 1987; 1c, Bal. 2127; 2c, Bal. 2555; 4c, Bal. 8283.

Anthemis arvensis L. s.l. - 2c, Bal. 2007; 48p, Bal. 8253; 12p, Bal. 8777.

Anthemis arvensis L. subsp. arvensis - 43c, Bal. 2074.

Anthemis brachmannii Boiss. \& Heldr. - 33p, Bal. 8423.

This finding fills the gap between the populations of this species in northern and southern Peloponnisos already reported by Halácsy (1902).

Anthemis chia L. - 2c, Bal. 1802; 1c, Bal. 2073; 4c, Bal. 8278; 32o, Bal. s.n.

Anthemis cotula L. - 5p, Bal. 8189; 33p, Bal. 8421.

Bellis perennis L. - 2c, Bal. 1791; 7n, Bal. 8064.

Bellis sylvestris Cirillo - 30a, Bal. 2136.

Calendula arvensis L. - 41s, Bal. 6197; 34p, Bal. 8532; 38r, Bal. obs.

Carduus pycnocephalus L. - 37r, Bal. 2647; 5r, Bal. 8185.

Carthamus lanatus subsp. baeticus (Boiss. \& Reut.) Nyman - 29r, Bal. 2609.

Centaurea calcitrapa L. - 3r, Bal. 8293.

Centaurea pichleri Boiss. - 2c, Bal. 1794; 1c, Bal. 2081.

Centaurea raphanina subsp. mixta (DC.) Runemark - 2c, Bal. 1796; ibid., Bal. 2597; 4c, Bal. 8356.

Centaurea solstitialis L. subsp. solstitialis - 43p, Bal. 2080; 2c, Bal. 2598.

Chondrilla juncea L. - 28r, Bal. 2146; 7n, Bal. 8057.

Cichorium intybus L. $-34 \mathrm{p}$, Bal. 8546.

Cirsium vulgare (Savi) Ten. - 2c, Bal. 2603; 29r, Bal. 2608.

Crepis dioscoridis L. - 27q, Bal. 8609.

Crepis foetida L. subsp. foetida - 5p, Bal. 8191; 34p, Bal. 8482.

Crepis fraasii Sch. Bip. subsp. fraasii-5a, Bal. 8183; 34a, Bal. 8529; 12d, Bal.8741; 10g, Bal. 8793.

Crepis neglecta subsp. graeca (Vierh.) Rech. f. - 2c, Bal. 1793; 43c, Bal. 2077; 31r, Bal. 6142a; 5a, Bal. 8188; 4c, Bal. 8277.

Crepis rubra L. - 2c, Bal. 1799; 3c, Bal. 1986; 5p, Bal. 8192; 8r, Bal. s.n.; 4c, Bal. 8285. 
Crepis sancta (L.) Bornm. - 2c, Bal. 1792; 43c, Bal. 2076; 31r, Bal. 6142b; 4c, Bal. 8282.

Crepis setosa Haller f. - 28r, Bal. 2627.

Crepis zacintha (L.) Loisel. - 33p, Bal. 8420.

Crupina crupinastrum (Moris) Vis. - 2c, Bal. 2569; 4c, Bal. s.n.

Cynara cardunculus L. subsp. cardunculus - 37r, Bal. 2616.

Dittrichia viscosa (L.) Greuter s.l. - 26r, Bal. obs.

Echinops sphaerocephalus L. subsp. sphaerocephalus - 30r, Bal. 2615.

[Erigeron sumatrensis Retz.] - 28s, Bal. 2139.

Filago arvensis L. - 42p, Bal. 2098; 12p, Bal. 8769.

Filago eriocephala Guss. - 48p, Bal. 8252.

Filago gallica L. - 48p, Bal. 8254; 12p, Bal. 8768.

Filago pyramidata L. - 48p, Bal. 8217; 30n, Bal. 8578; 12p, Bal. 8770.

Galactites tomentosus Moench - 30o, Bal. 8560.

Hedypnois rhagadioloides (L.) F.W. Schmidt subsp. rhagadioloides - 5r, Bal. 8184; 33p,

Bal. 8422; 12p, Bal. 8707.

Helminthotheca echioides (L.) Holub - 37r, Bal. 2648.

Hypochaeris cretensis (L.) Bory \& Chaub. - 2c, Bal. 1961; 7n, Bal. 8055; 5p, Bal. 8190; 4c, Bal. 8290; 34p, Bal. 8526.

Inula verbascifolia (Willd.) Hausskn. s.l. - 34i, Bal. 8557; 26q, Bal. 8615.

Lactuca viminea subsp. ramosissima (All.) Arcang. - 1c, Bal. 2075.

Lapsana communis subsp. adenophora (Boiss.) Rech. f. - 12p, Bal. 8738.

Leontodon crispus subsp. asper (Waldst. \& Kit.) Rohlena - 2c, Bal. 1798.

Leontodon tuberosus L. - 30r, Bal. 2137; 32r, Bal. s.n.; 49r, Bal. obs.

Matricaria chamomilla L. - 19s, Bal. 2163; 7r, Bal. 8059.

Notobasis syriaca (L.) Cass. - 18r, Bal. obs.

Onopordum illyricum subsp. cardunculus (Boiss.) Arènes - 3r, Bal. 2602; 29r, Bal. 2612;

7r, Bal. obs.; 14r, Bal. obs.

Pallenis spinosa (L.) Cass. subsp. spinosa - 33p, Bal. 8425.

Phagnalon rupestre subsp. graecum (Boiss. \& Heldr.) Batt. - 34i, Bal. 8466; 27q, Bal. 8604.

Picnomon acarna (L.) Cass. - 2c, Bal. 2599; 6r, Bal. obs.; 29r, Bal. obs.; 27r, Bal. obs. Picris pauciflora Willd. - 9r, Bal. 8817.

Picris rhagadioloides (L.) Desf. - 32r, Bal. 2636; 33p, Bal. 8424; 34p, Bal. 8480; 26r, Bal. 8624.

Pilosella bauhini (Schult.) Arv.-Touv. s.l. - 12d, Bal. 8705.

Pilosella cymosa subsp. sabina (Sebast.) H.P. Fuchs - 4c, Bal. 8287.

Podospermum canum C.A. Mey. - 2c, Bal. 1797; 7r, Bal. 8038; 4c, Bal. 8280.

Ptilostemon afer (Jacq.) Greuter subsp. afer - 29r, Bal. 2613.

Ptilostemon chamaepeuce (L.) Less. - 34i, Bal. 8468.

Ptilostemon stellatus (L.) Greuter - 37r, Bal. 2040; 30r, Bal. 2614; 33p, Bal. 8446; 30o, Bal. 8559; 26r, Bal. 8623; 12p, Bal. 8708; 48p, Bal. s.n.

Pulicaria odora (L.) Rchb. - 12d, Bal. 8717.

Reichardia picroides (L.) Roth - 31r, Bal. 6145; 27r, Bal. 8607.

Rhagadiolus stellatus (L.) Gaertn. -37r, Bal. 1768; 32o, Bal. 6125; 7n, Bal. 8060; 6k, Bal. 8132; 34p, Bal. 8481. 
All the specimens were initially determined as Rhagadiolus edulis Gaertn. which according to Dimopoulos \& al. (2013) is a synonym.

Scolymus hispanicus L. subsp. hispanicus - 30r, Bal. 2611; 151, Bal. obs.

Scorzonera crocifolia Sm. - 9r, Bal. 8798.

Scorzoneroides cichoriacea (Ten.) Greuter - 12p, Bal. 8726.

Senecio vernalis Waldst. \& Kit. - 2c, Bal. 1800; 46p, Bal. obs.

Senecio vulgaris L. - 2c, Bal. 1801; 4c, Bal. 8279; 41s, Bal. obs.; 45s, Bal. obs.

Silybum marianum (L.) Gaertn. - 7r, Bal. obs.; 6n, Bal. obs.; 151, Bal. obs.; 26r, Bal.obs.; 17r, Bal. obs.

Sonchus asper subsp. glaucescens (Jord.) Ball - 31r, Bal. 6146.

Tragopogon porrifolius subsp. eriospermus (Ten.) Greuter - 30r, Bal. 6154; 9r, Bal. 8799.

Tragopogon samaritani Heldr. \& Sartori ex Boiss. - 2c, Bal. 2596; 4c, Bal. 8288.

Urospermum picroides (L.) F.W. Schmidt - 48p, Bal. 8234.

[Xanthium spinosum L.] - 31r, Bal. 6141.

Xeranthemum inapertum (L.) Mill. - 2c, Bal. 1795.

\section{BETULACEAE}

Carpinus orientalis Mill. -40a, Bal. 6171; 5a, Bal. 8167; 34a, Bal. 8553; 22r, Bal. 8636; 10g, Bal. 8786.

Ostrya carpinifolia Scop. - 34a, Bal. 8478.

\section{BORAGINACEAE}

Alkanna methanaea Hausskn. - 48p, Bal. 8231.

Anchusa azurea Mill. - 23r, Bal. obs.

Anchusa undulata subsp. hybrida (Ten.) Bég. - 2c, Bal. 1837; 42p, Bal. 2082; 32r, Bal. 6133.

Anchusella cretica (Mill.) Bigazzi, Nardi \& Selvi - 2c, Bal. 1838; 32o,r, Bal. 6114; 39a, Bal. 6168; 7r, Bal. s.n.; 4c, Bal. s.n.; 44s, Bal. obs.; 49r, Bal. obs.

Buglossoides incrassata (Guss.) I.M. Johnst. subsp. incrassata - 2c, Bal. 1841; 4c, Bal. 8368.

Cerinthe major L. - 30r, Bal. 6151.

Cerinthe retorta $\mathrm{Sm} .-34 \mathrm{p}$, Bal. 8472.

Cynoglossum columnae Ten. - 7n, Bal. 8097; 44r, Bal. obs.

Cynoglossum creticum Mill. - 2c, Bal. 1836; 7n, Bal. 8096.

Echium italicum subsp. biebersteinii (Lacaita) Greuter \& Burdet - 7r, Bal. obs.; 49r, Bal. obs.; 15r, Bal. obs.; 28r, Bal. obs.; 26r, Bal. obs.

Echium plantagineum L. -48p, Bal. 8229; 30o, Bal. 8564; 14r, Bal. obs.

Heliotropium europaeum L. - 28s, Bal. 2141.

Myosotis ramosissima Rochel subsp. ramosissima - 2c, Bal. 1839.

Myosotis sylvatica subsp. cyanea (Hayek) Vestergren - 2c, Bal. 1840; 5a, Bal. 8149; 4c, Bal. 8369.

Onosma frutescens Lam. - 34i, Bal. 8501; 27q, Bal. 8613.

Symphytum bulbosum K.F. Schimp. - 7k, Bal. 8095; 48k, Bal. 8230; 12d, Bal. s.n.; 6k, Bal. obs. 


\section{BRASSICACEAE}

Aethionema saxatile subsp. graecum (Boiss. \& Spruner) Hayek - 2c, Bal. 1831; 39q, Bal. 6159; 4b, Bal. 8306; 31a, Bal. s.n.

Alliaria petiolata (M. Bieb.) Cavara \& Grande-40k, Bal. 6185; 7k, Bal. 8091; 41s, Bal. obs.; 36k, Bal. obs.; 47s, Bal. obs.

Alyssum foliosum Bory \& Chaub. - 43c, Bal. 2126; 2c, Bal. 2553; 4c, Bal. 8302.

Alyssum montanum subsp. repens (Baumg.) Schmalh. - 2c, Bal. 1835.

Alyssum murale Waldst. \& Kit. - 2c, Bal. 1966.

Alyssum siculum Jord. - 2c, Bal. 1828; 4c, Bal. 8300.

Alyssum simplex Rudolphi - 32r, Bal. 6128; 28r, Bal. 8587; 14r, Bal. 8669; 9r, Bal. 8820. Alyssum strigosum Banks \& Sol. - 2c, Bal. 2547.

Arabis sagittata (Bertol.) DC. - 34q, Bal. 8544.

Arabis turrita L. - 34q, Bal. 8471.

Arabis verna (L.) R.Br. - 2c, Bal. 1829; 4c, Bal. 8298.

Aurinia saxatilis subsp. orientalis (Ard.) T.R. Dudley - 32r, Bal. 2022; 34i, Bal. 8485; 28r, Bal. 8586; 27q, Bal. 8599 .

Bunias erucago L. - 32o, Bal. 6120.

Calepina irregularis (Asso) Thell. - 32o,r, Bal. 6119; 41s, Bal. s.n.; 35r, Bal. obs.; 47r, Bal. obs.; 38r, Bal. obs.

Capsella bursa-pastoris (L.) Medik. - 2c, Bal. 1847; 32o, Bal. 6121; 48p, Bal. 8241; 4c, Bal. 8295; 41s, Bal. s.n.; 47s, Bal. obs.

Cardamine graeca L. -6k, Bal. 8130.

Cardamine hirsuta L. - 2c, Bal. 2275; 40r, Bal. 6187; 48p, Bal. 8214.

Clypeola jonthlaspi L. subsp. jonthlaspi - 2c, Bal. 1832; 39a, Bal. 6169; 32r, Bal. s.n.

Draba muralis L. - 41t, Bal. 6189; 4c, Bal. 8294.

Draba praecox Steven - 2c, Bal. 2276; 40r, Bal. 6186.

Draba verna L. - 4c, Bal. 8307.

Erysimum asperulum Boiss. \& Heldr. - 48p, Bal. 8250.

Erysimum pectinatum Bory \& Chaub. - 4c, Bal. 8305.

Hirschfeldia incana (L.) Lagr.-Foss. - 7r, Bal. 8092; 33p, Bal. s.n.

Hornungia petraea (L.) Rchb. - 4c, Bal. 8303.

Isatis tomentella Boiss. \& Balansa - 27q, Bal. 8600.

Lepidium coronopus (L.) Al-Shehbaz - 19u, Bal. 2511.

Lepidium draba L. subsp. draba-47s, Bal. obs.

Lepidium graminifolium L. - 28s, Bal. 2140.

Lepidium hirtum subsp. nebrodense (Raf.) Thell. - 2c, Bal. 1830.

Lunaria annua subsp. pachyrhiza (Borbás) Maire \& Petitm. - 34p, Bal. 8470.

Malcolmia graeca subsp. bicolor (Boiss. \& Heldr.) Stork - 2c, Bal. 1834; 43c, Bal. 2056;

4c, Bal. 8297.

Microthlaspi perfoliatum (L.) F.K. Mey. - 2c, Bal. 1833; 30r, Bal. s.n.; 43c, Bal. s.n.

Nasturtium officinale R. Br. - 35m, Bal. 6111; 34m, Bal. 8515.

Rapistrum rugosum (L.) All. - 28r, Bal. 8838.

Rorippa sylvestris (L.) Besser subsp. sylvestris - 19u, Bal. 2160.

Sisymbrium officinale (L.) Scop. - 2c, Bal. 1846; 4c, Bal. 8299.

Sisymbrium orientale L. - 4c, Bal. 8296; 45s, Bal. obs. 


\author{
CAESALPINIACEAE \\ Cercis siliquastrum L. - 34a, Bal. s.n.; 7k, Bal. obs.; 26a, Bal. obs.
}

\title{
CAMPANULACEAE
}

Asyneuma limonifolium (L.) Janch. subsp. limonifolium - 43c, Bal. 2070.

Campanula ramosissima Sm. - 37r, Bal. 1771; 2c, Bal. 2558; 28s, Bal. 2621; 7r, Bal. 8061; 5p, Bal. 8205; 28t, Bal. 8592; 10p, Bal. 8796; 12p, Bal. s.n.

Campanula spatulata subsp. spruneriana (Hampe) Hayek - 2c, Bal. 1951; 5a, Bal. 8204; 4c, Bal. 8322.

Campanula versicolor Andrews - 21q, Bal. obs.

Legousia falcata (Ten.) Fritsch ex Janch. - 28r, Bal. 8836.

Legousia hybrida (L.) Delarbre - 2c, Bal. 1861; 39r, Bal. 6161; 4c, Bal. 8324.

Legousia speculum-veneris (L.) Chaix - 2c, Bal. 2006; 5a, Bal. 8169.

\section{CAPRIFOLIACEAE}

Sambucus nigra L. - 15n, Bal. 8644.

\section{CARYOPHYLLACEAE}

Arenaria guicciardii Heldr. ex Boiss. - 43c, Bal. 2063; 2c, Bal. 2540; 4c, Bal. 8321.

A Greek endemic which is rare and scattered in the mountains of Kriti, southern parts of mainland, Ionian and E Aegean islands (Phitos 1997). Its finding in various localities of Mt Aphrodisio and Mt Likeo (Baliousis 2013), particularly in their upper altitudinal zone, extends considerably its distribution in Peloponnisos.

Arenaria leptoclados (Rchb.) Guss. - 2c, Bal. 2542; 7r, Bal. 8106; 4c, Bal. 8319.

Arenaria serpyllifolia L. - 43c, Bal. 2064.

Cerastium brachypetalum subsp. roeseri (Boiss. \& Heldr.) Nyman - 2c, Bal. 1857; 43c, Bal. 2066; 32r, Bal. 6130; 31r, Bal. 6135a; 4c, Bal. 8312.

Cerastium glomeratum Thuill. - 2c, Bal. 1856; 31r, Bal. $6135 b$.

Cerastium illyricum subsp. brachiatum (Lonsing) Jalas - 48p, Bal. 8240; 4c, Bal. 8314. Holosteum umbellatum L. - 2c, Bal. 2283.

Minuartia globulosa (Labill.) Schinz \& Thell. - 2c, Bal. 2536; 9r, Bal. 8804.

Minuartia hamata (Hausskn. \& Bornm.) Mattf. -43c, Bal. 2058; 4c, Bal. 8316.

Minuartia hybrida (Vill.) Schischk. - 5p, Bal. 8180.

Minuartia mesogitana (Boiss.) Hand.-Mazz. s.l. - 4c, Bal. 8315.

Petrorhagia dubia (Raf.) G. López \& Romo - 39r, Bal. 6157; 48p, Bal. 8239; 4c, Bal. 8318; 12p, Bal. 8757.

Petrorhagia glumacea (Bory \& Chaub.) P.W. Ball \& Heywood - 32r, Bal. 2017; 2c, Bal. 2571; 33p, Bal. 8462; 30r, Bal. 8571; 9r, Bal. 8803.

Petrorhagia illyrica (L.) P.W. Ball \& Heywood subsp. illyrica-43c, Bal. 2062; 2c, Bal. 2564.

Polycarpon tetraphyllum (L.) L. - 34p, Bal. 8551.

Saponaria calabrica Guss. - 3q, Bal. 1855; 43p, Bal. 2067; 7r, Bal. 8108; 32r, Bal. s.n.; 39q, Bal. s.n.; 46q, Bal. obs.

Scleranthus verticillatus Tausch - 4c, Bal. 8310.

Silene conica L. - 4c, Bal. 8320. 
Silene cretica L. - 2c, Bal. 1854; 4c, Bal. 8323.

Silene gigantea subsp. hellenica Greuter - 34p, Bal. 8490; 27q, Bal. 8605.

Silene italica subsp. peloponnesiaca Greuter - 2c, Bal. 1949; 2e, Bal. 2563; 12d, Bal. 8718.

Silene nocturna L. - 28s, Bal. 8835.

Silene nutabunda Greuter - 26q, Bal. 8630.

This is the northernmost locality of this species endemic to Peloponnisos.

Silene vulgaris subsp. macrocarpa Turrill - 7n, Bal. 8107.

Silene vulgaris (Moench) Garcke s.l. - 31q, Bal. 6148; 9r, Bal. 8809.

Stellaria apetala Ucria - 2c, Bal. 1859; 42p, Bal. 2061; 32o, Bal. 6122.

Stellaria cupaniana Jord. \& Fourr. - 28s, Bal. 2265; 39r, Bal. 6162.

Stellaria media (L.) Vill. - 30n, Bal. 6152.

Velezia rigida L. $-9 \mathrm{q}$, Bal. 8805.

CHENOPODIACEAE

Chenopodium vulvaria L. - 19u, Bal. 2162.

CISTACEAE

Cistus creticus subsp. eriocephalus (Viv.) Greuter \& Burdet - 5a, Bal. 8166; 14r, Bal. 8691; 12d, Bal. 8727.

Cistus salviifolius L. $-12 \mathrm{~d}$, Bal. 8728.

Fumana arabica (L.) Spach - 26q, Bal. 8621.

Fumana thymifolia (L.) Webb - 26q, Bal. 8620.

Helianthemum nummularium (L.) Mill. subsp. nummularium - 2c, Bal. 1862; 5a, Bal. 8187; 31a, Bal. s.n.

Helianthemum salicifolium (L.) Mill. - 2c, Bal. 1863; 39r, Bal. 6158; 4c, Bal. 8395.

Tuberaria guttata (L.) Fourr. - 48p, Bal. 8218.

\section{CONVOLVULACEAE}

Calystegia silvatica (Kit.) Griseb. - 37r, Bal. 2026; 12d, Bal. 8764.

Convolvulus arvensis L. - 1c, Bal. 2068; 7o, Bal. s.n.; 4c, Bal. s.n.

Convolvulus cantabrica L. - 32r, Bal. 1849; 2c, Bal. 1970; 30r, Bal. 8566; 39r, Bal. s.n.; 33p, Bal. s.n.

Convolvulus elegantissimus Mill. - 2c, Bal. 1971; 2e, Bal. 2524; 4c, Bal. 8365.

\section{CRASSULACEAE}

Sedum amplexicaule subsp. tenuifolium (Sm.) Greuter - 43c, Bal. 2107; 2c, Bal. 2549; 7k, Bal. 8083; 4c, Bal. 8383; 12d, Bal. 8719; 22d, Bal. s.n.

Sedum caespitosum (Cav.) DC. - 4c, Bal. 8384.

Sedum cepaea L. - 28m, Bal. 2628; 34i, Bal. 8475.

Sedum hispanicum L. - 2b, Bal. 1945; 27q, Bal. 8601.

Sedum laconicum Boiss. \& Heldr. subsp. laconicum - 2b, Bal. 1946; 43c, Bal. 2125; 4b, Bal. 8382.

Sedum rubens L. - 37r, Bal. 1947; 7n, Bal. 8084; 30r, Bal. 8413; 14r, Bal. 8688.

Umbilicus chloranthus Heldr. \& Sartori ex Boiss. - 26t, Bal. 8629. 
Umbilicus horizontalis (Guss.) DC. - 4b, Bal. 8388; 34i, Bal. 8494.

Umbilicus rupestris (Salisb.) Dandy - 34q, Bal. 8523.

\section{CUCURBITACEAE}

Bryonia cretica L. - 28r, Bal. 2617.

Ecballium elaterium (L.) A. Rich. - 26s, Bal. obs.

\section{DIPSACACEAE}

Cephalaria ambrosioides (Sm.) Roem. \& Schult. - 27q, Bal. 8612.

Knautia integrifolia (L.) Bertol. s.l. -2c, Bal. 1848; 32r, Bal. 2640; 7n, Bal. 8098; 6n, Bal. 8116; 5p, Bal. 8203; 48p, Bal. 8249; 4c, Bal. 8391; 12p, Bal. 8767.

Pterocephalus plumosus (L.) Coult. - 33p, Bal. 8433.

\section{ERICACEAE}

Arbutus unedo L. - 36j, Bal. obs.

Erica arborea L. - 36j, Bal. obs.

\section{EUPHORBIACEAE}

Euphorbia apios L. - 2c, Bal. 1884; 4c, Bal. 8380.

Euphorbia helioscopia L. - 2c, Bal. 1883; 32o, Bal. 6115.

Euphorbia peplus L. - 32r, Bal. 6131.

Mercurialis annua L. - 39r, Bal. s.n.; 45t, Bal.obs.; 28t, Bal.obs.; 26t, Bal. obs.; 15t, Bal. obs.

\section{FABACEAE}

Anthyllis vulneraria subsp. rubriflora (DC.) Arcang. - 2c, Bal. 1813; 42p, Bal. 2113; 5a, Bal. 8157; 9r, Bal. 8826.

Astragalus depressus L. subsp. depressus - 2c, Bal. 1825; 1c, Bal. 2128; 4c, Bal. 8350. Astragalus glycyphyllos subsp. glycyphylloides (DC.) Maire \& Petitm. - 22r, Bal. 8635; $12 \mathrm{p}$, Bal. 8750.

Astragalus hamosus L. - 37r, Bal. 2046; 5r, Bal. 8150.

Bituminaria bituminosa (L.) C.H. Stirt. - 31r, Bal. 6144; 14r, Bal. obs.; 29r, Bal. obs.; 26r, Bal. obs.

Calicotome villosa (Poir.) Link - 34a, Bal. 8502; 36j, Bal. obs.; 31a, Bal. obs.; 27a, Bal. obs.

Chamaecytisus hirsutus (L.) Link s.l. - 40r, Bal. 6175.

Coronilla scorpioides (L.) W.D.J. Koch - 2c, Bal. 1821; 30r, Bal. 8410; 33p, Bal. 8453; 9r, Bal. s.n.

Dorycnium herbaceum Vill. - 42p, Bal. 2118; 12p, Bal. 8723; 22r, Bal. obs.

Dorycnium hirsutum (L.) Ser. - 37r, Bal. 2037; 34q, Bal. 8538; 22r, Bal. obs.

Hippocrepis biflora Spreng. - 30r, Bal. 8411; 9r, Bal. 8825.

Hippocrepis emerus subsp. emeroides (Boiss. \& Spruner) Greuter \& Burdet ex Lassen 2c, Bal. 1822; 40a, Bal. 6174; 34a, Bal. 8497; 36j, Bal. obs.; 32a, Bal. obs.

Hymenocarpos circinnatus (L.) Savi - 7r, Bal. 8071; 30r, Bal. 8402; 22r, Bal. 8641; 31r, Bal. s.n.; 32r, Bal. s.n.; 12p, Bal. s.n. 
Lathyrus amphicarpos L. - 2c, Bal. 1817.

Lathyrus aphaca L. - 37r, Bal. 1762; 42p, Bal. 2110; 2c, Bal. 2532; 14r, Bal. 8672b; 30r, Bal. s.n.

Lathyrus digitatus (M. Bieb.) Fiori - 2c, Bal. 1811; 9a, Bal. 8831.

Lathyrus laxiflorus (Desf.) Kuntze - 40a, Bal. 6179; 5a, Bal. 8158; 22d, Bal. s.n.

Lathyrus niger (L.) Bernh. - 12p, Bal. 8763.

Lathyrus pratensis L. - 14m, Bal. 8650.

Lathyrus setifolius L. -47r, Bal. 6201.

Lathyrus sphaericus Retz. - 14r, Bal. 8671; 12p, Bal. 8702; 9q, Bal. 8830.

Lens ervoides (Brign.) Grande - 37r, Bal. 1763; 34p, Bal. 8548; 12p, Bal. 8701.

Lotus angustissimus L. - 48p, Bal. 8219; 12p, Bal. 8753.

Lotus conimbricensis Brot. - 30r, Bal. 8568; 14r, Bal. 8679.

Lotus longisiliquosus R. Roem. - 32r, Bal. 2012; 26r, Bal. 8627.

Lotus ornithopodioides L. - 30r, Bal. 8409; 9r, Bal. 8829; 37r, Bal. s.n.; 32o, Bal. s.n.

Lotus tenuis Willd. - 32r, Bal. 2011; 42m, Bal. 2111; 14m, Bal. 8647.

Lupinus albus subsp. graecus (Boiss. \& Spruner) Franco \& P.Silva - 22r, Bal. 8639.

Medicago arabica (L.) Huds. - 37r, Bal. s.n.; 7o, Bal. 8067; 6n, Bal. 8129; 5r, Bal. 8179;

8r, Bal. s.n.; 32o, Bal. s.n.

Medicago coronata (L.) Bartal. - 27r, Bal. 8603; 26q, Bal. 8622.

Medicago lupulina L. - 37r, Bal. 1761; 7r, Bal. 8072; 3r, Bal. 8352; 14r, Bal. 8651; 22r, Bal. s.n.

Medicago minima (L.) Bartal. - 37r, Bal. s.n.; 2c, Bal. 1812; 7r, Bal. 8068; 6n, Bal. 8119; 5r, Bal. 8163; 4c, Bal. 8351; 30r, Bal. 8407; 34p, Bal. 8510; 31r, Bal. s.n.; 48p, Bal. s.n.; 33p, Bal. s.n.; 30r, Bal. s.n.; 12p, Bal. s.n.; 9r, Bal. s.n.

Medicago monspeliaca (L.) Trautv. - 4c, Bal. 8390.

Medicago orbicularis (L.) Bartal. - 2c, Bal. 1818; 5p, Bal. 8160; 4c, Bal. s.n.

Medicago polymorpha L. - 6n, Bal. 8118; 5r, Bal. 8162; 34p, Bal. 8498; 14r, Bal. 8681;

10p, Bal. 8797; 48p, Bal. s.n.; 30o, Bal. s.n.; 12p, Bal. s.n.

Medicago rigidula (L.) All. - 2c, Bal. 1820; 7r, Bal. 8069; 5a, Bal. 8161; 48p, Bal. 8227; 4c, Bal. 8354; 12p, Bal. s.n.

Medicago sativa subsp. falcata (L.) Arcang. - 2c, Bal. 1979; 28s, Bal. 8594.

[Medicago sativa L. subsp. sativa] - 13r, Bal. 8783.

Melilotus graecus (Boiss. \& Spruner) Lassen - 42p, Bal. 2121; 33p, Bal. 8456; 9r, Bal. 8828; 26q, Bal. obs.

Melilotus indicus (L.) All. - 14r, Bal. 8649.

Melilotus italicus (L.) Lam. - 34p, Bal. 8477.

Melilotus neapolitanus Ten. - 42p, Bal. 2117; 33p, Bal. 8459; 34p, Bal. 8514.

Onobrychis aequidentata (Sm.) d'Urv. - 33p, Bal. 8461; 26q, Bal. 8617.

Onobrychis alba subsp. pentelica (Hausskn.) Nyman - 2c, Bal. 1819.

Onobrychis caput-galli (L.) Lam. - 37r, Bal. 1766b; 48p, Bal. 8225; 12p, Bal. 8703; 30r, Bal. s.n.

Ononis spinosa subsp. antiquorum (L.) Arcang. - 32r, Bal. 2018; 2c, Bal. 2529.

Ononis viscosa subsp. breviflora (DC.) Nyman - 33p, Bal. 8460.

Ornithopus compressus L. - 14r, Bal. 8678; 12p, Bal. s.n.

Scorpiurus muricatus L. - 30r, Bal. 8401; 34p, Bal. s.n.; 9r, Bal. s.n. 
Securigera cretica (L.) Lassen - 30r, Bal. 8400; 33p, Bal. 8451.

Securigera securidaca (L.) Degen \& Dörfl. - 37r, Bal. 1765; 30r, Bal. 8399; 27r, Bal. 8597.

Spartium junceum L. - 48e, Bal. obs.; 36r, Bal. obs.

Tetragonolobus purpureus Moench - 30n, Bal. 6150.

Trifolium angustifolium L. - 42p, Bal. 2114; 6n, Bal. 8123; 33p, Bal. 8457; 34p, Bal. 8549.

Trifolium arvense L. - 12d, Bal. 8697.

Trifolium aurantiacum Boiss. \& Spruner - 2c, Bal. 1816; 43p, Bal. 2115; 5a, Bal. 8154; 4c, Bal. s.n.; 12p, Bal. s.n.

Trifolium campestre Schreb. - 37r, Bal. 1766a; 34p, Bal. 8513; 14r, Bal. 8674; 12p, Bal. 8730; 43p, Bal. s.n.; 4c, Bal. s.n.

Trifolium cherleri L. - 3c, Bal. 1975; 5r, Bal. 8152; 48p, Bal. 8221; 4c, Bal. 8347; 30r, Bal. 8405; 34p, Bal. s.n.

Trifolium dalmaticum Vis. - 37r, Bal. 2028.

Trifolium glomeratum L. - 12d,p, Bal. 8734; ibid., Bal. 8749.

Trifolium grandiflorum Schreb. - 2c, Bal. 1815; 43p, Bal. 2116; 5a, Bal. 8153; 4c, Bal. 8353.

Trifolium hirtum All. - 12p, Bal. 8700.

Trifolium lappaceum L. - 32r, Bal. 2019; 33p, Bal. 8458; 30m, Bal. 8567.

Trifolium leucanthum M. Bieb. - 5a, Bal. 8151; 4c, Bal. 8343; 12p, Bal. 8698; 9q, Bal. 8823 .

Trifolium nigrescens Viv. - 37m, Bal. 1760; 3c, Bal. 1972a; 5r, Bal. 8155; 48p, Bal. 8224; 4c, Bal. 8345.

Trifolium ochroleucon subsp. roseum (C. Presl) Lassen - 22r, Bal. 8640; 12d, Bal. 8694.

Trifolium pallidum Waldst. \& Kit. - 37m, Bal. 2030; 19p, Bal. 2518; 7r, Bal. 8065; 48p, Bal. 8223; 33p, Bal. 8455; 12p, Bal. 8732; 14r, Bal. s.n.

Trifolium patens Schreb. - 14m, Bal. 8645 .

Trifolium physodes M. Bieb. - 37m, Bal. 1757; 2c, Bal. 1809; 2e, Bal. 1978; 39a, Bal. 6167; 4c, Bal. s.n.; 30a, Bal. s.n.; 34p, Bal. s.n.

Trifolium pignantii Fauché \& Chaub. - 22d, Bal. 8633; 12p, Bal. 8693.

Trifolium pratense L. - 37m, Bal. 1759; 42m, Bal. 2119; 28m, Bal. 2626.

Trifolium repens L. - 3c, Bal. 1972b; 37m, Bal. 2027; 42m, Bal. 2120; 7o, Bal. 8066.

Trifolium resupinatum L. subsp. resupinatum - 19u, Bal. 2510; 34m, Bal. 8518; 30m, Bal. 8576; 14r, Bal. 8673.

Trifolium scabrum L. - 5r, Bal. 8156; 48p, Bal. 8226.

Trifolium stellatum L. - 2c, Bal. 1814; 5p, Bal. 8164; 4c, Bal. 8344; 30r, Bal. 8406; 33p, Bal. 8454; 48p, Bal. s.n.

Trifolium striatum L. - 2c, Bal. 1977; 4c, Bal. 8348; 12p, Bal. 8733; 9q, Bal. 8822.

Trifolium subterraneum L. - 37r, Bal. 1758; 2c, Bal. 1810; 6n, Bal. 8125; 8r, Bal. s.n.; 48p, Bal. 8228; 4c, Bal. 8346; 12p, Bal. 8755; 12d, Bal. s.n.

Trifolium tenuifolium Ten. -48p, Bal. 8220; 14f, Bal. 8675.

Trifolium tomentosum L. - 5p, Bal. 8206; 34p, Bal. 8509.

Trigonella corniculata (L.) L. s.l. - 42p, Bal. 2122; 9r, Bal. 8827.

Trigonella gladiata M. Bieb. - 2c, Bal. 2561; 9r, Bal. 8821. 
Tripodion tetraphyllum (L.) Fourr. - 33p, Bal. 8450.

Vicia angustifolia L. - 31r, Bal. 6140; 5p, Bal. 8159; 34p, Bal. 8517; 14r, Bal. 8646.

Vicia hybrida L. - 37r, Bal. 1764; 2c, Bal. 1827; 39r, Bal. 6160.

Vicia laeta Ces. - 22r, Bal. 8634.

Vicia lathyroides L. - 2c, Bal. 1824; 4c, Bal. 8355; 12p, Bal. 8780.

Vicia lutea L. subsp. lutea - 14r, Bal. $8672 a$.

Vicia melanops Sm. - 28r, Bal. 8833.

Vicia parviflora Cav. - 9q, Bal. 8819.

Vicia villosa subsp. eriocarpa (Hausskn.) P.W. Ball - 42p, Bal. 2112; 30n, Bal. 6156; 6n, Bal. 8117; 14r, Bal. 8648 .

\section{FAGACEAE}

Quercus coccifera L. - 39a, Bal. obs.; 4c, Bal. s.n.; 30a, Bal.obs.; 36j, Bal. obs.; 32a, Bal. obs.; 31a, Bal. obs.; 40a, Bal. obs.; 46a, Bal. obs.; 5a, Bal. obs.; 9a, Bal. obs.; 29a, Bal. obs.; 27a, Bal. obs.; 22a, Bal. obs.; 16a, Bal. obs.

Quercus frainetto Ten. - 22d, Bal. 8632; 14f, Bal. 8689; 12d, Bal. 8716.

Quercus ilex L. - 34a, Bal. 8496.

Quercus pubescens Willd. - 2e, Bal. 1878; 48e, Bal. 8257; 4c, Bal. 8386; 14f, Bal. 8690; 9a, Bal. obs.; 27a, Bal. obs.; 22a, Bal. obs.

\section{FUMARIACEAE}

Corydalis solida subsp. incisa Lidén - 2b, Bal. 2287.

Fumaria officinalis L. subsp. officinalis - 2c, Bal. 1864; 28t, Bal. 8591.

\section{GENTIANACEAE}

Blackstonia perfoliata (L.) Huds. subsp. perfoliata - 34p, Bal. 8533.

Centaurium erythraea Raf. subsp. erythraea - 12p, Bal. 8714.

Centaurium tenuiflorum (Hoffmanns. \& Link) Fritsch subsp. tenuiflorum - 32r, Bal. 2013.

\section{GERANIACEAE}

Erodium cicutarium (L.) L'Hér. - 2c, Bal. 1869; 43c, Bal. 2069; 5p, Bal. 8175; 4c, Bal. 8358.

Erodium malacoides (L.) L'Hér. - 31r, Bal. 6143.

Geranium asphodeloides Burm. f. subsp. asphodeloides - 6k, Bal. 8128; 12d, Bal. 8722. Geranium brutium Gasp. - 32o, Bal. 6116; 36r, Bal. obs.; 41r, Bal. obs.; 7r, Bal. obs.; 37r, Bal. obs.; 39o, Bal. obs.; 45s, Bal. obs.

Geranium columbinum L. - 31a, Bal. 6139; 39r, Bal. 6163; 6k, Bal. 8126; 7k, Bal. s.n.; 12p, Bal. s.n.; 9r, Bal. s.n.

Geranium dissectum L. - 37m, Bal. 1777; 32o, Bal. 6117; 12p, Bal. 8758; 7m, Bal. s.n. Geranium lucidum L. - 2b, Bal. 1871; 32t, Bal. 6124; 4b, Bal. s.n.; 47t, Bal. obs.

Geranium macrostylum Boiss. - 2b, Bal. 1872; 4b, Bal. 8361.

Geranium molle L. - 31r, Bal. 6147; 6n, Bal. 8127; 4c, Bal. 8359; 5p, Bal. s.n.

Geranium purpureum Vill. - 37r, Bal. 1778; 32r, Bal. 6129; 30r, Bal. s.n.

Geranium rotundifolium L. - 7r, Bal. 8051; 45s, Bal. obs. 
HYPERICACEAE

Hypericum perforatum L. s.l. - 32r, Bal. 2634; 14r, Bal. 8684.

Hypericum perfoliatum L. - 33p, Bal. 8473.

\section{LAMIACEAE}

Acinos graveolens (M. Bieb.) Link - 2c, Bal. 1845; 9r, Bal. 8806.

Acinos suaveolens (Sm.) Loudon - 43p, Bal. 2060.

Ballota acetabulosa (L.) Benth. - 3c, Bal. 1989; 4c, Bal. 8336.

Calamintha nepeta subsp. glandulosa (Req.) P.W. Ball - 28r, Bal. 2134.

Clinopodium vulgare subsp. orientale Bothmer -42p, Bal. 2124; 2e, Bal. 2567; 5a, Bal. 8186; 12d, Bal. 8729.

Lamium amplexicaule L. - 2c, Bal. 1843; 41s, Bal. 6191.

Lamium bifidum Cirillo subsp. bifidum - 28s, Bal. 2263; 41s, Bal. obs.

Lamium garganicum subsp. striatum (Sm.) Hayek - 2b, Bal. 1842; 4b, Bal. 8337.

Marrubium vulgare L. - 7n, Bal. 8103; 151, Bal. obs.

Melissa officinalis subsp. altissima (Sm.) Arcang. - 32o, Bal. 6126; 7k, Bal. 8102; 34m, Bal. 8521; 12p, Bal. 8737.

Mentha spicata subsp. condensata (Briq.) Greuter \& Burdet -32m, Bal. 2635; 7m, Bal. 8105. Micromeria juliana (L.) Rchb. - 32a, Bal. 2021; 2b, Bal. 2575; 33p, Bal. 8449; 26q, Bal. 8619; 5a, Bal. s.n.; 30a, Bal. s.n.; 9r, Bal. s.n.

Origanum vulgare subsp. hirtum (Link) A. Terracc. - 19r, Bal. 2164; 3c, Bal. 2572; 7r, Bal. 8104. Phlomis fruticosa L. - 36r, Bal. obs.; 32n, Bal. obs.; 46a, Bal. obs.; 8a, Bal. obs.; 27a, Bal. obs. Phlomis samia L. - 4c, Bal. 8339; 12d, Bal. 8712; 9a, Bal. obs.

Prunella laciniata (L.) L. - 43p, Bal. 2057; 3c, Bal. 2527; 6r, Bal. 8143; 5p, Bal. 8148; 12p, Bal. 8724.

Salvia fruticosa Mill. - 34a, Bal. 8491.

Salvia pomifera subsp. calycina (Sm.) Hayek - 32q, Bal. 2020; 27q, Bal. 8595.

Salvia verbenaca L. - 41s, Bal. 6192; 26r, Bal. 8614; 13r, Bal. 8785; 9r, Bal. 8818.

Salvia verticillata L. subsp. verticillata -33p, Bal. 8438; 26r, Bal. 8616.

Salvia virgata Jacq. - 32r, Bal. 1844.

Salvia viridis L. - 30n, Bal. 8572.

Scutellaria rupestris subsp. parnassica (Boiss.) Greuter \& Burdet - 30r, Bal. 8414; 33p, Bal. 8448 .

Sideritis purpurea Talbot ex Benth. - 7r, Bal. 8101; 33q, Bal. 8439; 9q, Bal. 8807.

Stachys graeca Boiss. \& Heldr. - 9r, Bal. 8808.

Stachys spinulosa Sm. - 37r, Bal. 1769; 33p, Bal. 8436.

Teucrium capitatum L. subsp. capitatum - 2c, Bal. 2573; 48p, Bal. 8251; 33p, Bal. s.n.; 30r, Bal. s.n.

Teucrium chamaedrys L. subsp. chamaedrys - 2c, Bal. 2574; 4c, Bal. 8338; 26q, Bal. 8618.

Teucrium flavum subsp. hellenicum Rech. f. - 33q, Bal. 8437.

Teucrium scordium L. subsp. scordioides (Schreb.) Arcang. - 34m, Bal. 8516.

Thymbra capitata (L.) Cav. - 36r, Bal. 2645.

Thymus longicaulis subsp. chaubardii (Rchb. f.) Jalas - 2c, Bal. 1953; 43c, Bal. 2053; 4c, Bal. 8340 . 


\section{LAURACEAE}

Laurus nobilis L. - 34a, Bal. 8535.

\section{LINACEAE}

Linum bienne Mill. - 28r, Bal. s.n.

Linum corymbulosum Rchb. - 2c, Bal. 2543; 30r, Bal. 8412; 33p, Bal. 8426.

Linum pubescens subsp. sibthorpianum (Margot \& Reut.) P.H. Davis - 33p, Bal. 8427; 12 p, Bal. 8735 .

\section{LORANTHACEAE}

Loranthus europaeus Jacq. - 2e, Bal. 2523 parasitize on Quercus pubescens.

\section{LYTHRACEAE}

Lythrum junceum Banks \& Sol. - 37m, Bal. 1770.

MALVACEAE

Alcea biennis subsp. cretica (Weinm.) Valdés - 32r, Bal. 2631.

Malva neglecta Wallr. - 5r, Bal. 8165.

Malva setigera Schimp. \& Spenn. - 33p, Bal. 8432; 9q, Bal. 8811.

Malva sylvestris L. - 28r, Bal. 8837; 24r, Bal. obs.; 14r, Bal. obs.

Malva unguiculata (Desf.) Alef. - 34p, Bal. 8484.

MOLLUGINACEAE

Glinus lotoides L. - 19u, Bal. 2159.

This is a rare species in Greece and particularly in Peloponnisos where it has been registered only in one locality near Kalamata (Strid \& Tan 1997, dot maps).

\section{MORACEAE}

Ficus carica L. subsp. carica - 28s, Bal. obs.; 26s, Bal. obs.

\section{NYCTAGINACEAE}

[Mirabilis jalapa L.] - 28s, Bal. obs.

\section{OLEACEAE}

Fraxinus ornus L. - 40a, Bal. 6173; 34a, Bal. 8495; 32a, Bal. obs.; 22a, Bal. obs.; 12d, Bal. obs.

Phillyrea latifolia L. - 2e, Bal. 2521; 40a, Bal. 6182; 34a, Bal. 8540; 5a, Bal. s.n.; 31a, Bal. obs.; 9a, Bal. obs.; 29a, Bal. obs.; 27a, Bal. obs.; 22a, Bal. obs.

ONAGRACEAE

Epilobium lanceolatum Sebast. \& Mauri - 19m, Bal. 2165.

OROBANCHACEAE

Bellardia latifolia (L.) Cuatrec. subsp. latifolia - 31r, Bal. 6134. 
Orobanche minor Sm. - 7r, Bal. 8053.

Phelipanche nana (Reut.) Soják - 7r, Bal. 8054.

\section{PAPAVERACEAE}

Papaver apulum Ten. - 4c, Bal. 8364.

Papaver rhoeas L. - 2c, Bal. 1882; 26s, Bal. 8628; 14r, Bal. 8664.

\section{PLANTAGINACEAE}

Plantago afra L. - 31r, Bal. 6137; 33p, Bal. 8463; 27r, Bal. 8602.

Plantago bellardii All. subsp. bellardii-48p, Bal. 8216; 12p, Bal. 8773.

Plantago lagopus L. subsp. lagopus - 31r, Bal. 6138; 34p, Bal. 8476.

Plantago lanceolata L. -2c, Bal. 1867; 19u, Bal. 2519; 5r, Bal. 8176; 30m, Bal. 8562.

\section{PLATANACEAE}

Platanus orientalis L. - 41k, Bal. obs.; 7k, Bal. obs.; 36k, Bal. obs.; 48k, Bal. obs.; 49k, Bal. obs.; 28k, Bal. obs.

\section{PLUMBAGINACEAE}

Armeria canescens (Host) Boiss. - 2c, Bal. 1948.

Plumbago europaea L. - 28r, Bal. 2132.

\section{POLYGALACEAE}

Polygala monspeliaca L. - 2c, Bal. 2556.

\section{POLYGONACEAE}

Polygonum arenastrum Boreau -28s, Bal. 2145.

Rumex bucephalophorus L. subsp. bucephalophorus - 12p, Bal. 8771.

Rumex conglomeratus Murray - 37m, Bal. 2035; 42m, Bal. 2092; 28m, Bal. 2629.

Rumex conglomeratus x Rumex pulcher-37m, Bal. 2033.

Rumex cristatus DC. - 28m, Bal. 8585.

Rumex obtusifolius L. s.l. $-37 \mathrm{~m}$, Bal. 1776.

Rumex pulcher L. subsp. pulcher - 4c, Bal. 8357.

Rumex pulcher L. s.l. - 19u, Bal. 2516; 7r, Bal. 8086.

Rumex tuberosus L. subsp. tuberosus - 2c, Bal. 1954; 4c, Bal. 8393.

Rumex tuberosus L. s.l. - 2c, Bal. 1876.

\section{PORTULACACEAE}

Portulaca oleracea L. s.l. - 28s, Bal. 2143.

\section{PRIMULACEAE}

Anagallis arvensis L. - 37r, Bal. s.n.; 33p, Bal. 8447; 12p, Bal. 8776.

Cyclamen hederifolium Sol. ex Aiton s.l. - 30a, Bal. 2147; 34a, Bal. 8555; 40a, Bal. s.n.; 7k, Bal. s.n.; 12d, Bal. s.n.

Lysimachia atropurpurea L. - 36r, Bal. 1850; 33p, Bal. 8428; 14r, Bal. obs.

Samolus valerandi L. $-34 \mathrm{~m}$, Bal. 8519. 


\section{RANUNCULACEAE}

Anemone apennina subsp. blanda (Schott \& Kotschy) Nyman - 2c,e, Bal. 2286; 40a, Bal. 6184; 46a, Bal. obs.

Clematis vitalba L. - 28r, Bal. 2620; 7k, Bal. s.n.

Consolida ajacis (L.) Schur - 8a, Bal. 8210; 30n, Bal. 8570; 13r, Bal. 8784.

Delphinium hellenicum Pawl. - 30r, Bal. 2652.

Ficaria cf. ficarioides (Bory \& Chaub.) Halácsy - 2c, Bal. 2278.

$F$. ficarioides is generally distinct from $F$. verna, but apparently intermediate plants have been observed, e.g., on Mt Klokos in N Peloponnisos, and it is possible they hybridise in areas of contact (Strid 2002). It seems that the above mentioned specimen belongs to this category.

Ficaria verna Huds. s.l. - 2c, Bal. 2277.

Helleborus odorus subsp. cyclophyllus (A. Braun) Maire \& Petitm. - 2e, Bal. 2302.

To my knowledge this is the southernmost locality of this Balkan endemic.

Nigella damascena L. - 2c, Bal. 2010; 7n, Bal. 8062; 33p, Bal. 8464; 8r, Bal. s.n.

Ranunculus chius DC. - 34p, Bal. 8528 .

Ranunculus gracilis E.D. Clarke - 2c, Bal. 1873; 39a, Bal. 6166; 40q, Bal. 6176; 5a, Bal. 8181.

Ranunculus muricatus L. - 34m, Bal. 8527; 30r, Bal. 8577.

Ranunculus neapolitanus Ten. - 37m, Bal. 1767; 22r, Bal. 8638; 9r, Bal. 8810.

Ranunculus paludosus Poir. - 28r, Bal. 8834.

Ranunculus psilostachys Griseb. - 2c, Bal. 1874; 4c, Bal. 8371.

Ranunculus sardous Crantz - 19u, Bal. 2512; 35m, Bal. 6112; 7m, Bal. 8063.

Ranunculus sprunerianus Boiss. - 2c, Bal. 1875.

Ranunculus velutinus Ten. - 6k, Bal. 8133; 12d, Bal. 8742; 10g, Bal. 8789.

\section{RHAMNACEAE}

Paliurus spina-christi Mill. - 37r, Bal. 2045.

Rhamnus saxatilis subsp. prunifolia (Sm.) Aldén - 4c, Bal. 8375.

\section{ROSACEAE}

Agrimonia eupatoria L. subsp. eupatoria - 12d, Bal. 8715.

Aremonia agrimonoides (L.) DC. s.l. -40a, Bal. 6177; 5a, Bal. 8168; 22d, Bal. 8637; 12d, Bal. 8740 .

Crataegus heldreichii Boiss. - 2c, Bal. 1877; 1c, Bal. 2083; 4c, Bal. 8374.

Crataegus monogyna Jacq. - 2e, Bal. 2607; 39a, Bal. 6165; 12d, Bal. 8711.

Geum urbanum L. - 6k, Bal. 8124.

Potentilla micrantha DC. - 2e, Bal. 2301.

Potentilla reptans L. - 19u, Bal. 2517; 14m, Bal. 8663.

Prunus mahaleb L. - 7k, Bal. 8100; 22d, Bal. 8631.

Pyrus spinosa Forssk. - 2e, Bal. 2605; 36r, Bal. obs.; 46n, Bal. obs.; 48e, Bal. obs.

Rosa canina L. - 12d, Bal. 8761.

Rosa pulverulenta M. Bieb. - 1c, Bal. 2059.

Rosa sempervirens L. - 37r, Bal. 2034. 
Rubus sanctus Schreb. - 28r, Bal. 2625.

Sanguisorba minor subsp. balearica (Nyman) Muñoz Garm. \& C. Navarro - 37r, Bal. s.n.

\section{RUBIACEAE}

Crucianella angustifolia L. - 1c, Bal. 2106; 2c, Bal. 2570; 4c, Bal. 8367; 12p, Bal. s.n. Crucianella latifolia L. - 37r, Bal. 1789b; 34p, Bal. 8488.

Cruciata laevipes Opiz - 42p, Bal. 2096; 7r, Bal. 8099; 45s, Bal. obs.

Cruciata pedemontana (Bellardi) Ehrend. - 2c, Bal. 1805.

Galium aparine L. - 7n, Bal. 8112; 28r, Bal. 8588.

Galium capitatum Bory \& Chaub. - 2c, Bal. 1808.

Galium intricatum Margot \& Reut. - 2c, Bal. 1957; 7r, Bal. 8111; 5a, Bal. 8170; 34p, Bal. 8520 .

Galium murale (L.) All. - 28t, Bal. $8590 b$.

Galium peloponnesiacum Ehrend. \& Krendl-2c, Bal. 1806; 2c, Bal. 1955; 43c, Bal. 2094. Galium tenuissimum M. Bieb. - 43c, Bal. 2091.

Galium verticillatum Danthoine - 2b, Bal. 1804; 43c, Bal. 2072; 4b, Bal. 8389.

Galium verum L. subsp. verum - 8r, Bal. 8209; 14r, Bal. 8666.

Rubia peregrina L. - 34a, Bal. 8554.

Sherardia arvensis L. - 37r, Bal. 1789a; 2c, Bal. 1807; 31r, Bal. s.n.; 32o, Bal. s.n.; 7n, Bal. s.n.; 6n, Bal. s.n.; 5p, Bal. s.n.; 8r, Bal. s.n.; 48p, Bal. s.n.; 4c, Bal. s.n.; 33p, Bal. s.n.; 9r, Bal. s.n.

Theligonum cynocrambe L. - 7r, Bal. 8094; 32o, Bal. s.n.; 41t, Bal. s.n.; 34p, Bal. s.n.; 28t, Bal. obs.

\section{SALICACEAE}

Salix alba L. - 14m, Bal. 8661.

\section{SANTALACEAE}

Osyris alba L. - 36j, Bal. 1772.

\section{SAXIFRAGACEAE}

Saxifraga hederacea L. - 41t, Bal. 6188.

Saxifraga rotundifolia L. s.l. - 4b, Bal. 8362.

Saxifraga tridactylites L. - 47t, Bal. 6205.

\section{SCROPHULARIACEAE}

Scrophularia canina subsp. bicolor (Sm.) Greuter - 7r, Bal. 8056; 44r, Bal. obs.; 29r, Bal. obs.; 27r, Bal. obs.

Scrophularia peregrina L. - 47s, Bal. 6207.

Verbascum daenzeri (Fauché \& Chaub.) Kuntze - 12q, Bal. 8739.

Verbascum macrurum Ten. - 30r, Bal. 2150; 43p, Bal. obs.; 29r, Bal. obs.

SIMAROUBACEAE

[Ailanthus altissima (Mill.) Swingle] - 15r, Bal. obs.; 17r, Bal. obs. 


\section{ULMACEAE}

Ulmus minor Mill. subsp. minor - 48k, Bal. 6198.

\section{URTICACEAE}

Parietaria judaica L. - 45t, Bal. obs.; 28t, Bal. obs.; 26t, Bal. obs.; 15s, Bal.obs.

Urtica dioica L. - 28r, Bal. 2618; 7k, Bal. 8113; 4c, Bal. 8392; 41n, Bal. obs.; 44s, Bal. obs.; 47n, Bal. obs.; 6k, Bal. obs.

\section{VALERIANACEAE}

Centranthus ruber subsp. sibthorpii (Boiss.) Hayek - 34i, Bal. 8499.

Valeriana italica Lam. - 2b, Bal. 1860; 9q, Bal. 8814.

Valerianella dentata (L.) Pollich - 48p, Bal. 8256; 34p, Bal. 8534.

Valerianella discoidea (L.) Loisel. - 2c, Bal. 1880.

Valerianella echinata (L.) DC. - 2c, Bal. 1879; 4c, Bal. 8331.

Valerianella eriocarpa Desv. - 9r, Bal. 8816.

Valerianella turgida (Steven) Betcke - 2c, Bal. 1881; 4c, Bal. 8330.

\section{VERBENACEAE}

Verbena officinalis L. $-28 \mathrm{~m}$, Bal. 8584.

\section{VERONICACEAE}

[Antirrhinum majus L. s.l.] - 44s, Bal. obs.; 28s, Bal. obs.; 26s, Bal. obs.

[Cymbalaria muralis G. Gaertn., B. Mey. \& Scherb. subsp. muralis] - 28t, Bal. 8590a.

Digitalis laevigata subsp. graeca (Ivanina) Werner - 5p, Bal. 8182; 34p, Bal. 8489.

Kickxia elatine subsp. crinita (Mabille) Greuter - 30r, Bal. 8569; 26r, Bal. 8625.

Linaria pelisseriana (L.) Mill. - 48p, Bal. 8232.

Linaria simplex Desf. - 4c, Bal. 8332.

Veronica anagallis-aquatica L. subsp. anagallis-aquatica-35m, Bal. 6113; 34m, Bal. 8531.

Veronica arvensis L. - 32r, Bal. 6132; 41s, Bal. 6193; 5r, Bal. 8171.

Veronica chamaedrys subsp. chamaedryoides (Bory \& Chaub.) M.A. Fisch. - 12d, Bal. 8713; 10g, Bal. 8790.

Veronica cymbalaria Bodard - 47t, Bal. 6206.

Veronica glauca subsp. chaubardii (Boiss. \& Reut.) Maire \& Petitm. - 2c, Bal. 1865; 43c, Bal. 2079; 4c, Bal. 8335.

Veronica hederifolia L. - 2c, Bal. 2281.

[Veronica persica Poir.] - 28r, Bal. 2229; 32o, Bal. 6118; 41s, Bal. 6195.

Veronica triloba (Opiz) Opiz - 41s, Bal. 6194.

\section{VIOLACEAE}

Viola kitaibeliana Schult. - 2c, Bal. 1888.

Viola odorata L. - 2e, Bal. 2300.

Viola phitosiana Erben - 2c, Bal. 2299.

ZYGOPHYLLACEAE

Tribulus terrestris L. - 28s, Bal. 2142. 


\section{MONOCOTYLEDONES}

ALISMATACEAE

Alisma plantago-aquatica L. - 37m, Bal. 2032.

ALLIACEAE

Allium amethystinum Tausch - 34p, Bal. 8465; 27q, Bal. 8596.

Allium ampeloprasum L. - 32r, Bal. 2633; 13r, Bal. 8782.

Allium chamaespathum Boiss. - 30a, Bal. 2148.

Allium flavum subsp. tauricum (Besser ex Rchb.) K. Richt. - 2c, Bal. 2568.

Allium guttatum subsp. tenorei (Parl.) Soldano - 2c, Bal. 2565.

Allium subhirsutum L. subsp. subhirsutum - 34a, Bal. 8492.

AMARYLLIDACEAE

Galanthus reginae-olgae subsp. vernalis Kamari-2e, Bal. 2298.

Sternbergia lutea (L.) Spreng. subsp. lutea-30r, Bal. 2129; 2c, Bal. 2294.

ARACEAE

Arum italicum Mill. subsp. italicum - 7k, Bal. 8037.

ASPARAGACEAE

Asparagus acutifolius L. - 2e, Bal. 2520; 30a, Bal. 6153; 7k, Bal. 8087.

\section{ASPHODELACEAE}

Asphodeline lutea (L.) Rchb. - 34i, Bal. 8500.

Asphodelus ramosus L. subsp. ramosus - 37m, Bal. 1775; 32r, Bal. 2643; 39a, Bal. 6170; 30n, Bal. 8565; 14r, Bal. obs.

All the above mentioned specimens belong to var. nervosus (Pomel) Z. Díaz \& Valdés. Their large fruits, $10-12 \times 8-9 \mathrm{~mm}$ (Bal. 2643), 9-10 x 7-9 mm (Bal. 1775) meet the size expected in A. nervosus Pomel (= A. messeniacus Heldr. ex Halácsy). Var. nervosus occurs also in Mt Likeo (Bal. 6110, with fruits $9-10 \times 8 \mathrm{~mm}$ and length of tepals up to 13-14 mm). It covers also the area between the two mountains (Bal. obs.). These specimens confirm the expected distribution of this variety in Greece (Díaz Lifante, in litt.). The fruits are larger than those in specimens from Mt Pendelikon (Baliousis 2011) in Attiki, with fruits of 5.5-7.5 $\times 4-7 \mathrm{~mm}$, which correspond to the var. ramosus $(=A$. microcarpus Viv.), as it was delimited in the revision of the genus Asphodelus (Díaz Lifante \& Valdés 1996).

\section{CYPERACEAE}

Carex distachya Desf. - 2c, Bal. 1901.

Carex distans L. $-14 \mathrm{~m}$, Bal. 8655.

Carex flacca subsp. serrulata (Spreng.) Greuter-37m, Bal. 1782; 2c, Bal. 2594; 12d, Bal. 8731.

Carex otrubae Podp. - 37m, Bal. 2042; 14m, Bal. 8652.

Cyperus fuscus L. - 19u, Bal. 2158. 
Cyperus longus L. s.l. - 37m, Bal. 2043; 32m, Bal. 2638; 28m, Bal. 8580.

Scirpoides holoschoenus (L.) Soják - 37m, Bal. 2044; 42m, Bal. 2095; 32m, Bal. 2642;

28m, Bal. 8582; 14m, Bal. 8654 .

DIOSCOREACEAE

Dioscorea communis (L.) Caddick \& Wilkin - 6k, Bal. 8131; 10p, Bal. 8792.

\section{HYACINTHACEAE}

Bellevalia dubia subsp. boissieri (Freyn) Feinbrun - 2c, Bal. 1853; 32r, Bal. s.n.

Drimia numidica (Jord. \& Fourr.) J.C. Manning \& Goldblatt - 31r, Bal. obs.; 39a, Bal. obs.; 36r, Bal. obs.; 27r, Bal. obs.

Muscari comosum (L.) Mill. - 7r, Bal. 8088; 4c, Bal. 8325.

Muscari neglectum Guss. ex Ten. - 2c, Bal. 1852; 30r, Bal. s.n.

Ornithogalum collinum Guss. subsp. collinum - 30a, Bal. 2256; 32o, Bal. s.n.; 39o, Bal. obs. Ornithogalum fimbriatum subsp. gracilipes (Zahar.) Landström - 2c, Bal. 2293; 4c, Bal. 8329.

Ornithogalum montanum Ten. - 2c, Bal. 1851; 4c, Bal. 8328.

Ornithogalum nutans L. - 4c, Bal. 8327.

Ornithogalum prasinantherum Zahar. - 14r, Bal. 8692.

Ornithogalum sibthorpii Greuter - 30a, Bal. 2231.

Prospero autumnale (L.) Speta - 30a, Bal. 2131.

Scilla nivalis L. s.l. - 2c,e, Bal. 2289.

\section{IRIDACEAE}

Crocus cancellatus subsp. mazziaricus (Herb.) B. Mathew - 2c, Bal. 2295; 4c, Bal. 8341. Crocus hadriaticus Herb. - 30r, Bal. 2130.

Crocus nivalis Bory \& Chaub. - 2c,e, Bal. 2297.

Crocus olivieri J. Gay subsp. olivieri-2c, Bal. 2296.

Gladiolus italicus Mill. - 32n, Bal. 8839.

[Iris germanica L.] - 28n, Bal. obs.

Iris tuberosa L. - 30a, Bal. obs.

Iris unguicularis subsp. carica (Wern. Schultze) A.P. Davis \& Jury - 30r, Bal. 2239; 40r, Bal. 6180.

Romulea linaresii subsp. graeca Bég. - 30a, Bal. 2230.

\section{JUNCACEAE}

Juncus bufonius L. - 30m, Bal. 8561; 14r, Bal. 8685.

Juncus inflexus L. - 42m, Bal. 2093; 34m, Bal. 8512; 28m, Bal. 8581; 14m, Bal. 8653.

Luzula forsteri (Sm.) DC. s.l. - 40a, Bal. 6183; 5a, Bal. 8177; 12d, Bal. 8721; 10g, Bal. 8788 .

\section{LILIACEAE}

Gagea amblyopetala Boiss. \& Heldr. - 2c, Bal. 2291.

Gagea bohemica (Zauschn.) Schult. \& Schult. f. - 2c, Bal. 2292.

Gagea graeca (L.) Irmisch - 36r, Bal. 6208.

Gagea villosa (M. Bieb.) Sweet - 2c, Bal. 2290. 


\section{ORCHIDACEAE}

Anacamptis coriophora subsp. fragrans (Pollini) R.M. Bateman, Pridgeon \& M.W. Chase - 30r, Bal. 8574.

Anacamptis laxiflora (Lam.) R.M. Bateman, Pridgeon \& M.W. Chase subsp. laxiflora 14r, Bal. 8665.

Anacamptis pyramidalis (L.) Rich. - 20r, Bal. 8642; 8r, Bal. obs.; 14r, Bal. obs.; 33p, Bal. obs.; 22r, Bal. obs.

Epipactis helleborine (L.) Crantz subsp. helleborine - 14f, Bal. 8670; 12d, Bal. 8704.

Ophrys lutea subsp. galilaea (H. Fleischm. \& Bornm.) Soó - 30a, Bal. obs.

\section{POACEAE}

Achnatherum bromoides (L.) P. Beauv. - 32a, Bal. 2641; 34p, Bal. 8541.

Aegilops biuncialis Vis. subsp. biuncialis - 2c, Bal. 2001a; ibid., Bal. 2587; 5p, Bal. 8198b; 4c, Bal. 8258b; 33p, Bal. 8443b.

Aegilops comosa Sm. subsp. comosa - 2c, Bal. 2001c; ibid., Bal. 2588; 6n, Bal. 8140a; 5p, Bal. 8198a; 33p, Bal. 8443a; 9r, Bal. 8832.

Aegilops markgrafii (Greuter) Hammer - 34p, Bal. 8508.

Aegilops neglecta Bertol. subsp. neglecta - 2c, Bal. 2586; 32r, Bal. 2637; 7n, Bal. 8047; 8a, Bal. 8211; 4c, Bal. 8258a; 33p, Bal. 8442; 12p, Bal. 8762 .

Aegilops triuncialis L. subsp. triuncialis - 2c, Bal. 2001b; ibid., Bal. 2589; 7n, Bal. 8046; 6n, Bal. 8140b; 33p, Bal. 8441; 12p, Bal. 8759.

Aira elegantissima Schur - 5a, Bal. 8194; 4c, Bal. 8309.

Alopecurus myosuroides Huds. $-41 \mathrm{~s}$, Bal. 6196.

Alopecurus rendlei Eig - 7r, Bal. 8045; 14m, Bal. 8660.

Anthoxanthum odoratum L. - 48k, Bal. 8245; 12d, Bal. 8720 .

Avena barbata Link subsp. barbata - 14r, Bal. 8657; 12p, Bal. 8747.

Avena sterilis subsp. ludoviciana (Durieu) Gillet \& Magne - 28r, Bal. 8589; 37r, Bal. s.n. Brachypodium glaucovirens (Murb.) Sagorski - 28m, Bal. 2623.

Brachypodium retusum (Pers.) P. Beauv. - 2c, Bal. 1998; 43c, Bal. 2087; 42c, Bal. 2102; 5a, Bal. s.n.; 4c, Bal. 8275; 34a, Bal. s.n.

Brachypodium sylvaticum (Huds.) P. Beauv. subsp. sylvaticum - 37m, Bal. 2041; 7k, Bal. 8041; 12d, Bal. 8709.

Briza humilis M. Bieb. - 4b, Bal. 8271.

Briza maxima L. - 37r, Bal. 1784; 7n, Bal. s.n.; 49r, Bal. obs.

Bromus alopecuros Poir. s.l. - 37r, Bal. 1787; 7r, Bal. 8040; 30r, Bal. 8417.

Bromus hordeaceus subsp. mediterraneus (H. Scholz \& F.M. Vázquez) H. Scholz - 2c, Bal. 1992.

Bromus intermedius Guss. subsp. intermedius - 37r, Bal. 1788; 43c, Bal. 2103; 2c, Bal. 2577; 5a, Bal. 8193; 4c, Bal. 8273; 12p, Bal. 8774.

Bromus madritensis L. s.l. - 2c, Bal. 1898; 5p, Bal. 8197.

Bromus parvispiculatus $\mathrm{H}$. Scholz - 2c, Bal. 1892.

A recently described species. In Peloponnisos it has been registered from a few localities of its northern parts. The species is certainly undercollected (Scholz 2008).

Bromus scoparius L. - 48p, Bal. 8236.

Bromus squarrosus L. subsp. squarrosus - 2c, Bal. 1994; 43c, Bal. 2086; 4c, Bal. 8274. 
Bromus sterilis L. - 2c, Bal. 1996; 43c, Bal. 2085; 7n, Bal. 8050; 48p, Bal. s.n.; 4c, Bal. 8269; 34p, Bal. 8506; 14r, Bal. 8659.

Bromus tectorum L. - 2c, Bal. 1899; 43p, Bal. 2054; 4c, Bal. 8264.

Catapodium rigidum (L.) C.E. Hubb. - 2c, Bal. 1997; 5p, Bal. 8195; 34p, Bal. 8543; 4c, Bal. s.n.; 33p, Bal. s.n.; 30r, Bal. s.n.

Crypsis alopecuroides (Piller \& Mitterp.) Schrad. - 19u, Bal. 2157.

This is the first record of this species from Peloponnisos.

Crypsis schoenoides (L.) Lam. - 19u, Bal. 2156.

Cynosurus echinatus L. -2c, Bal. 1897; 7n, Bal. 8039; 30r, Bal. 8418; 33p, Bal. s.n.; 34p,

Bal. s.n.; 30n, Bal. s.n.

Cynosurus effusus Link - 5a, Bal. 8200; 4c, Bal. 8276.

Dactylis glomerata subsp. hispanica (Roth) Nynan - 2c, Bal. 1900; 4c, Bal. 8262; 12d, Bal. s.n.

Dasypyrum villosum (L.) P. Candargy - 43p, Bal. 2084; 7n, Bal. 8044.

Festuca arundinacea Schreb. s.l. - 7k, Bal. 8049.

Festuca jeanpertii subsp. achaica (Markgr.-Dann.) Markgr.-Dann. - 2c, Bal. 1889; 1c, Bal. 2089; 43c, Bal. 2101.

Festuca jeanpertii (St.-Yves) Markgr. subsp. jeanpertii-2c, Bal. $2585 a$.

Gastridium sp. - 34p, Bal. 8530; 12p, Bal. 8746.

Gaudinia fragilis (L.) P. Beauv. - 37r, Bal. 1790; 32r, Bal. 2014b; 6n, Bal. 8142; 48p, Bal. 8244; 30r, Bal. 8419; 33p, Bal. 8444; 14r, Bal. 8658.

Gaudiniopsis macra (M. Bieb.) Eig s.l. - 42p, Bal. 2105.

Hainardia cylindrica (Willd.) Greuter - 32r, Bal. 2014a; 30r, Bal. 8416; 14r, Bal. 8686.

Helictochloa agropyroides (Boiss.) Romero Zarco - 4c, Bal. 8268.

Helictotrichon convolutum (C. Presl) Henrard - 2c, Bal. 2579.

Holcus lanatus L. subsp. lanatus - 42m, Bal. 2100; 32m, Bal. 2644.

Hordeum bulbosum L. - 2c, Bal. 2581; 6n, Bal. 8141.

Hordeum geniculatum All. - 7r, Bal. 8052.

Hordeum murinum subsp. leporinum (Link) Arcang. - 2c, Bal. 1891; 7r, Bal. 8043; 4c, Bal. 8265; 34p, Bal. 8556 .

Hyparrhenia hirta (L.) Stapf - 33p, Bal. 8440; 26r, Bal. 8626; 27r, Bal. obs.

Lolium multiflorum Lam. - 37m, Bal. 1785.

Lolium perenne L. - 7n, Bal. 8042.

Lolium cf. perenne L. - 19u, Bal. 2513.

Lolium rigidum Gaudin subsp. rigidum - 2c, Bal. 1999; 32r, Bal. s.n.; 37r, Bal. 2031b; 43c, Bal. 2088; 42p, Bal. 2104; 7n, Bal. 8077; 5p, Bal. 8201; 4c, Bal. 8266; 30r, Bal. 8415; 34p, Bal. 8507; 14r, Bal. 8687; 12p, Bal. 8748.

Melica ciliata L. subsp. ciliata - 2c, Bal. 1990; ibid., Bal. 2591.

Melica uniflora Retz. - 10g, Bal. 8787.

Phleum phleoides (L.) H. Karst. - 2c, Bal. 1893; 43c, Bal. 2090; 4c, Bal. 8259.

Piptatherum miliaceum (L.) Coss. s.l. $-28 \mathrm{r}$, Bal. obs.

Poa annua L. subsp. annua - 19m, Bal. 2514.

Poa bulbosa L. s.l. - 2c, Bal. 1894; 4c, Bal. 8261; 7n, Bal. s.n.; 12p, Bal. s.n.

Poa compressa L. - 2c, Bal. 2585 b. 
Poa timoleontis Heldr. ex Boiss. - 2c, Bal. 1890.

Poa trivialis subsp. sylvicola (Guss.) H. Lindb. -37m, Bal. 1786; 7k, Bal. 8048.

Polypogon monspeliensis (L.) Desf. - 37m, Bal. 2039; 34m, Bal. 8503.

Polypogon viridis (Gouan) Breistr. - 34m, Bal. 8537.

Psilurus incurvus (Gouan) Schinz \& Thell. - 31r, Bal. 6136; 5p, Bal. 8196; 48p, Bal. 8243; 12p, Bal. 8756.

Rostraria cristata (L.) Tzvelev - 48p, Bal. 8235; 34p, Bal. 8505.

[Setaria pumila (Poir.) Roem. \& Schult.] - 28s, Bal. 2152.

Stipa capensis Thunb. - 30r, Bal. 8579.

Stipa holosericea Trin. subsp. holosericea - 2c, Bal. 2005.

Trachynia distachya (L.) Link - 33p, Bal. 8445; 27r, Bal. 8598; 12p, Bal. 8779; 9r, Bal. s.n.

Vulpia ciliata Dumort. subsp. ciliata - 2c, Bal. 1895; 5p, Bal. 8199; 4c, Bal. 8263.

Vulpia myuros (L.) C.C. Gmel. - 2c, Bal. 2004; 12p, Bal. 8736.

\section{RUSCACEAE}

Ruscus aculeatus L. - 7k, Bal. 8089; 6k, Bal. obs.

\section{SMILACACEAE}

Smilax aspera L. - 34a, Bal. 8552; 25a, Bal. obs.

\section{Vegetation}

The largest part of the investigated area is covered by open scrub, macchie and deciduous Oak forests. Transitional vegetation types also exist depending on the intense of human influences and bioclimatic conditions.

The dominant vegetation type of the mountain is Quercus coccifera scrub in various stages of transition to dense impenetrable macchie. It covers mainly the lower altitudinal zone of the mountain $(500-1100 \mathrm{~m})$ predominantly on limestone. As they occupy the inhabited zone these formations have received great pressure by man especially in the past. Quercus coccifera is almost always accompanied by Phillyrea latifolia and frequently both species constitute a characteristic association. These formations are often interspersed with scattered individuals of deciduous elements such as Quercus pubescens, Fraxinus ornus, Crataegus spp., Acer monspessulanum subsp. monspessulanum, Carpinus orientalis and Pistacia terebinthus subsp. terebinthus the latter especially at lower altitudes. Open space of overgrazed units is often covered by Phlomis fruticosa dominated phrygana vegetation. This is more obvious in western parts of the mountain along the road which connects the villages Kardaritsi and Paralogi.

In places where topoclimatic and edaphic conditions are appropriate e.g. localities with more humid conditions and deeper soil Quercus pubescens, Carpinus orientalis and Fraxinus ornus form pure stands or mixed deciduous woods. These formations are indicators of local differentiations in ecological conditions. Quercus pubescens presents its optimum of growth in northern slopes of the mountain. In this case it alters perceptibly the physiognomy of Quercus coccifera- Phillyrea latifolia communities described earlier. Its cover increases with altitude while the proportion of sclerophyllus elements declines. Its 
abundance ranges from a few isolated individuals in $600 \mathrm{~m}$ to numerous vigorous and tall plants at approximately $1000 \mathrm{~m}$. In the latter zone Quercus pubescens forms open forests where Quercus coccifera constitutes the shrub layer.

Quercus frainetto forests locally intermixed with Quercus pubescens or Fraxinus ornus occupy a relatively large area in eastern parts of the mountain, mainly around Nasia village. Characteristic species of the underfloor are the following: Brachypodium sylvaticum, Crepis fraasii subsp. fraasii, Geranium asphodeloides subsp. asphodeloides, Veronica chamaedrys subsp. chamaedryoides, Epipactis helleborine subsp. helleborine, Ranunculus velutinus, Phlomis samia, Aremonia agrimonoides s.l., Pulicaria odora, Crataegus monogyna, Cyclamen hederifolium. Open spaces are characterized by Pteridium aquilinum subsp. aquilinum. The presence of many young individuals of Quercus frainetto indicates good rates of regeneration.

The upper zone of the mountain above 1100 or $1200 \mathrm{~m}$ is deforested. It is characterized by stony meadows which are occasionally interrupted by rocky outcrops. The meadows are interspersed with strongly browsed shrubs of Quercus coccifera, Crataegus heldreichii and solitary trees of Quercus pubescens. Characteristic species of the herb layer are the following: Scandix australis subsp. grandiflora, Alyssum siculum, Aethionema saxatile subsp. graecum, Ornithogalum montanum, Lamium garganicum subsp. striatum, Thymus longicaulis subsp. chaubardii, Veronica glauca subsp. chaubardii, Astragalus depressus subsp. depressus, Geranium macrostylum, Myosotis sylvatica subsp. cyanea, Corydalis solida subsp. incisa, Sedum amplexicaule subsp. tenuifolium, Ranunculus psilostachys, Eryngium amethystinum. It hosts also a significant number of Greek endemics relatively rare in the area such as the following: Geocaryum parnassicum, Cerastium illyricum subsp. brachiatum, Ornithogalum fimbriatum subsp. gracilipes, Aristolochia microstoma, Erysimum pectinatum, Sedum laconicum subsp. laconicum. Grass cover is relatively high and dominated by Festuca jeanpertii s.l., Phleum phleoides, Brachypodium retusum and Poa bulbosa. Patches with deeper soil host thick populations of herbaceous species such as Capsella bursa-pastoris, Trifolium stellatum, Trifolium nigrescens, Stellaria spp., Sisymbrium officinale, Geranium molle, Urtica dioica. Their floristic composition is similar to those of pastures in the lower altitudinal zone. It is obvious that one of the main ecological factors that have shaped the physiognomy of the upper part of the mountain is grazing by goats and sheep. Small occurrences of flysch in this zone are easily distinguished dy the predominance of Pteridium aquilinum subsp. aquilinum.

Vegetation units with Abies cephalonica are absent though this species is relatively abundant in the nearby Mt Lambia. There seems to be no restrictions related to bioclimatic or edaphic conditions and the upper parts of the mountain could potentially sustain such formations.

Finally, an extensive area with conglomerates in the lower altitudinal zone of the mountain $(200-500 \mathrm{~m})$ south of the village Voutsis bears a floristically differentiated type of macchie consisting of Quercus coccifera, Arbutus unedo, Erica arborea, Pistacia lentiscus and Calicotome villosa. The lower altitude, the geological substrate and the relatively high air humidity favored the development of this type of macchie.

Streams dispersed all over the investigated area are often lined by Platanus orientalis woods. Their floristic composition is strongly influenced by man as many of them cross inhabited areas. 


\section{Discussion}

According to the present investigation, 650 taxa were found to comprise the vascular flora of Mt Aphrodisio. Crypsis alopecuroides is a new record for Peloponnisos. The largest in number of taxa families are the following: Fabaceae (90), Asteraceae (73), Poaceae (69).

The endemic vascular flora consists of 29 taxa (4.5\%). It includes some rare or local taxa such as Geocaryum parnassicum, Aristolochia microstoma, Alkanna methanaea, Erysimum asperulum, Erysimum pectinatum, Anthemis brachmannii, Silene gigantea subsp. hellenica, Delphinium hellenicum, Galium capitatum, Verbascum daenzeri, Viola phitosiana. It seems that there is a higher proportion of Greek endemics in higher altitudinal zone as $11(37.9 \%)$ of them were found exclusively at altitudes above $1000 \mathrm{~m}$. There are only three regional endemics of Peloponnisos, Anthemis brachmannii, Erysimum pectinatum and Silene nutabunda, growing on the mountain. This is a characteristic difference with the flora of Mt Likeo (Baliousis 2013) which comprises nine taxa of this chorological category. The latter mountain is situated at about the same longitude but in southern Peloponnisos and as a result it includes a number of regional endemics with distribution confined to southern Peloponnisos. Both mountains have about the same size and the same geological history as they belong to the same geotectonic unit. Additionally, they have been investigated to the same extent by the same author. Thus their difference in number of endemics can presumably be attributed to the southern geographical position of $\mathrm{Mt}$ Likeo. Balkan endemics are represented by 22 taxa (3.4\%). The number of adventive taxa is rather small (11 taxa) as compared to the ones of mountains which include heavily urbanized areas such as Mt Pendelikon (Baliousis \& Yannitsaros 2011; Baliousis 2011).

The physiognomy of the vegetation is dominated by Quercus coccifera. The most important deciduous element is Quercus pubescens, a basic constituent of the vegetation types discerned in northern slopes. Finally Quercus frainetto forms pure or mixed forests in eastern parts of the mountain.

\section{Acknowledgements}

I am grateful to Prof. Emer. D. Phitos (Patras) for his encouragement to accomplish this work and for his valuable comments and suggestions on the preparation of the manuscript. I would like also to thank the following persons for determining or confirming the determination of specimens of my collections from Mt Aphrodisio: Prof. Z. Díaz Lifante (Sevilla - Asphodelus, Centaurium), Prof. F. Ehrendorfer (Vienna - Rubiaceae), Ass. Prof. O. Georgiou (Patras - Anthemis, Petrorhagia), Prof. G. Kamari (Patras - Minuartia), P. Lassen (Lund - Fabaceae), Prof. H. Scholz (Berlin - Poaceae), Prof. S. Snogerup (Lund - Epilobium, Polygonum, Rumex), Prof. D. Tzanoudakis (Patras - Allium) and Prof. G. Wagenitz (Göttingen - Filago arvensis).

\section{References}

Bagnouls, F. \& Gaussen, H. 1957: Les climats biologiques et leur classification. - Ann. Géogr. 66: 193-220.

Baliousis, E. 2013: Flora and vegetation of Mt Likeo (Peloponnisos, Greece). - Fl. Medit. 23: 1547. doi: 10.7320/FlMedit23.015 
- 2011: I chlorida kai i vlastisi tou orous Pendelikou [The flora and vegetation of Mount Pendelikon (East Attiki, Greece)]. - PhD Thesis, Athens.

— \& Yannitsaros, A. 2011: Vascular plant diversity of Mt Pendelikon (Sterea Ellas, Greece): a recent inventory reflecting contemporary dynamics. - Willdenowia 41(1): 151-165. doi: 10.3372/wi.41.41119

Davis, P. H. (ed.) 1965-85: Flora of Turkey and the East Aegean Islands, 1-9. - Edinburgh.

Díaz Lifante, Z. \& Valdés, B. 1996: Revisión del género Asphodelus L. (Asphodelaceae) en el Mediterráneo Occidental. - Boissiera 52: 5-189.

Dimopoulos, P., Raus, Th., Bergmeier, E., Constantinidis, Th., Iatrou, G., Kokkini, S., Strid, A. \& Tzanoudakis, D. (ed.) 2013: Vascular plants of Greece: An annotated checklist. - Berlin \& Athens.

Emberger, L. 1955: Une classification biogéographique des climats. - Recueil Trav. Lab. Bot. Geol. Zool. Univ. Fac. Sci. Montpellier 7: 3-43.

- 1959: Orientation actuelle au service de la C. G. V. de la cartographie physiologique appliqué. Bull. Serv. Carte Phytogéogr., Ser. B, 4(2).

Greuter, W., Burdet, H. M. \& Long, G. (ed.) 1984, 1986, 1989: Med-Checklist, 1, 3, 4. - Genève \& Berlin.

— \& Raab-Straube, E. von (ed.) 2008: Med-Checklist, 2. - Palermo \& Berlin.

Halácsy, E. de 1902: Conspectus florae graecae, 2. - Lipsiae.

IGME 1978: Geological map of Greece. Kertezi sheet; scale 1: 50000. - Athens.

Phitos, D. 1997: Arenaria L. - Pp. 158-168 in: Strid, A. \& Tan, K. (ed.) Flora hellenica, 1. Königstein.

Sauvage, C. 1963: Le quotient pluviothermique d'Emberger, son utilization et la representasion géographique de ses variations au Maroc. - Ann. Phys. Globe Météorol. Inst. Sci. Chérif. 20: 11-23.

Scholz, H. 2008: Some comments on the genus Bromus (Poaceae) and three new species. Willdenowia 38(2): 411-422.

Strid, A. 2002: Ranunculus L. - Pp. 38-69 in: Strid, A. \& Tan, K. (ed.) Flora hellenica, 2. - Ruggell. — \& Tan, K. (ed.) 1997: Flora hellenica, 1. - Königstein.

— \& - (ed.) 2002: Flora hellenica, 2. - Ruggell.

Tutin, T. G., Heywood, V. H., Burges, N. A., Moore, D. M., Valentine, D. H., Walters, S. M. \& Webb, D. A. (ed.) 1968-1980: Flora europaea, 2-5. - Cambridge.

—, Burges, N. A., Chater, A. O., Edmondson, J. R., Heywood, V. H., Moore, D. M., Valentine, D. H., Walters, S. M. \& Webb, D. A. (ed.) 1993: Flora europaea, ed. 2, 1. - Cambridge.

Address of the author:

Baliousis Evagelos,

Gortinias 2, Dionisos Attiki, 14569, Greece. E-mail: baliousisv@biol.uoa.gr 
